\title{
Thermodynamic analysis of modified teleparallel gravity involving higher-order torsion derivative terms
}

\author{
Saira Waheed ${ }^{1, \mathrm{a}}$, M. Zubair ${ }^{2, \mathrm{~b}}$ \\ ${ }^{1}$ Prince Mohammad Bin Fahd University, Khobar, Kingdom of Saudi Arabia \\ 2 Department of Mathematics, COMSATS University Islamabad, Lahore Campus, Lahore, Pakistan
}

Received: 17 February 2019 / Accepted: 20 March 2019 / Published online: 12 April 2019

(C) The Author(s) 2019

\begin{abstract}
The present study is elaborated to investigate the validity of thermodynamical laws in a modified teleparallel gravity based on higher-order derivative terms of torsion scalar. For this purpose, we consider spatially flat FRW model filled with perfect fluid matter contents. Firstly, we explore the possibility of existence of equilibrium as well as nonequilibrium picture of thermodynamics in this extended version of teleparallel gravity. Here, we present the first law and the generalized second law of thermodynamics (GSLT) using Hubble horizon. It is found that non-equilibrium description of thermodynamics exists in this theory with the presence of an extra term called as entropy production term. We also establish GSLT using the logarithmic corrected entropy. Further, by taking the equilibrium picture, we discuss validity of GSLT at Hubble horizon for two different $F$ models. Using Gibbs law and the assumption that temperature of matter within Hubble horizon is similar to itself, We use different choices of scale factor to discuss the GSLT validity graphically in all scenarios. It is found that the GSLT is satisfied for a specified range of free parameters in all cases.
\end{abstract}

\section{Introduction}

Astronomical probes of modern cosmology suggest a speedy expanding state of cosmos caused by a leading ingredient of obscure nature present in the matter contents of cosmos, labeled as dark energy (DE) [1-5]. Numerous attempts have been made by the researchers to investigate this mysterious component successfully. A list of proposed candidates for $\mathrm{DE}$ is available in literature which is based on one of the two different strategies namely modified matter source models [6-12] and modified gravitational theories [13-15]. On the basis of their applications to various cosmological issues,

\footnotetext{
a e-mail: swaheed@pmu.edu.sa

be-mails:mzubairkk@gmail.com; drmzubair@cuilahore.edu.pk
}

a detailed analysis of these candidates favors the modified gravitational theories as the most successful tool for discussing different stages of cosmic evolution. Some promising modified gravitational theories include Gauss-Bonnet theory and its extended versions [16-19], $f(R)$ theory [20-22] and its different generalizations involving minimal or nonminimal interactions between different fields (higher-order curvature correction terms, matter and scalar fields as well as torsion scalar) like $f(R, T)[23-29]$ and $f(R, T, Q)$ theories [30-33], scalar-tensor theories and its generalized versions [34-38] and the well-known teleparallel gravity with its different extensions [39-44].

Teleparallel gravity is regarded as one of the interesting alternative to Einstein's gravity (GR) in which torsional formulation provides the gravitational source instead of curvature scalar structure of GR [45-50]. This theory is labeled as TEGR (teleparallel equivalent of general relativity) and is determined by the Lagrangian density involving curvature less Weitzenböck connection instead of torsion less Levi-Civita connection along with the vierbein as a fundamental tool. A variety of extended versions of this theory have been presented in literature like $f(T)$ gravity where a generic function of torsion scalar replaces the simple torsion scalar term in the Lagrangian density [39-44]. In this respect, another different version of this theory has been proposed by Kofinas and Saridakis [51-54] where they introduced a new term $T_{G}$ called teleparallel equivalent to Gauss-Bonnet term and then further, they extended this theory to a more general case named as $f\left(T, T_{G}\right)$ theory. Another significant modification is considered by Harko et al. $[55,56]$ by including a non-minimal interaction of torsion scalar with matter field in the action. In this respect, another recent significant modification is $f(T, B)$ gravity [57], where the term $B$ is related to the divergence of torsion tensor and is termed as boundary term. This theory has been tested by applying on different cosmological issues and found to be very interesting $[58,59]$. Another extended version of teleparallel grav- 
ity has been proposed in literature [60] which is based on higher order derivative terms like $\nabla T$ and $\square T$. The basic motivation for the inclusion of such terms emerges from already proposed other generalized versions of $f(R)$ gravity where different higher order terms of Ricci scalars like $R_{\mu \nu} R^{\mu \nu}, R_{\mu \nu \alpha \beta} R^{\mu \nu \alpha \beta},(\nabla R)^{2}$ etc., or its interaction with scalar as well as matter fields are introduced in order to incorporate the quantum corrections $[61,62]$. Another idea behind it to formulate a fundamental gravity like string theory where such terms arise in lagrangian density or Kaluza-Klein theories for reducing dimensions or to work at scales close to plank scales in effective quantum gravity.

The description of thermodynamical picture of accelerating cosmos is regraded as one of the most interesting issues in today's cosmology. "The concepts of gravitation and thermodynamics are interlinked with each other" is a fundamental connection supported by some well-known results of thermodynamical study of black holes (BH). The BH thermodynamics suggests the BHs as thermodynamical systems where the terms like temperature and entropy are associated with the geometrical quantities such as surface gravity and horizon area, respectively [63-65]. In this respect, the first effort was made by Jacobson who used $T_{h} d \hat{S}_{h}=\delta \hat{Q}$ (Clausius relation) along with $S=\frac{A}{4 G}$ to derive the GR field equations by taking Rindler model into account $(\hat{Q}, \hat{S}, T$ are notations for energy flux, entropy and temperature, respectively) [66]. Gibbons and Hawking [67] also made an attempt to explore these fundamental characteristics of thermodynamics using de Sitter model. Frolov and Kofman [68] used flat quasi de-Sitter inflationary model of cosmos for investigating such a connection of gravity and thermodynamics. They concluded that the dynamical equations of Einstein gravity for Friedmann model can be formulated using $d E=T d S$ with a slowly rolling scalar field. It is seen that the Einstein field equations for FRW universe can be obtained from the first law of thermodynamics at the apparent horizon by making the use of relationships for Hawking temperature and entropy given by $T_{A}=\frac{1}{2 \pi \tilde{R}_{A}}$ and $S_{A}=\frac{A}{4 G}$, respectively, where $A$ denotes the horizon area. Later on, this connection was verified by Padmanabhan [69] for a general spherically symmetric spacetime. He found that the dynamical equations for the considered model can be expressed in the form $d E+P d V=T d S$. The question about the validity of such connection has been already investigated in various contexts like braneworld [70,71], Gauss-Bonnet gravity [72], the Lovelock gravity [73,74], $f(R)$ gravity [75-77] and scalar-tensor theory [78].

Karami and Abdolmaleki [79] discussed the validity of GSLT in $f(T)$ gravity using Hubble horizon and two viable models of $f(T)$ involving future singularities. They concluded that for present and early eras, the GSLT remains valid while for later eras, it will be satisfied for a specific value of torsion scalar. For $f(R, T)$ and $f\left(R, T, R_{\mu \nu} T^{\mu \nu}\right)$ theories, the study of thermodynamics has been carried out by Sharif and Zubair [80] where they checked its validity at apparent horizon in non-equilibrium perspective and they also formulated some possible constraints on the coupling parameter. For a general Gauss-Bonnet theory namely $f(G)$ gravity, Abdolmaleki and Najafi [81] used matter and radiation filled FRW geometry along with two different $f(G)$ models to examine the validity of GSLT at dynamical apparent horizon. Further this study has also been extended to the case of $f(R, G)$ theory [82]. The study of thermodynamical laws has been also presented by Bahamonde et al. $[58,58]$ in a new modified teleparallel theory which relates both $f(R)$ and $f(T)$ gravities by the equation $R=-T+B$, where $B$ is the boundary term. They found that this theory suggests the existence of non-equilibrium thermodynamics picture due to the presence of additional entropy production term. Further, by including the coupling of scalar field with torsion and boundary term, the validity of GSLT has been investigated at apparent horizon with and without including logarithmic corrected entropy relation [83].

In a recent paper [84], GSLT validity has been explored by Azizi and Borhani in a teleparallel gravity involving a nonminimal coupling of torsion and matter and obtained interesting results. The validity of GSLT has also been explored in $f\left(T, T_{G}\right)$ theory and the possible constraints on the coupling parameter in terms of recent cosmic parameters and power law solution has been found [85]. Sharif and Waheed [86] checked the validity of GSLT at Hubble, apparent, particle and event horizons in a scalar-tensor gravity involving chameleonic field as well as magnetic field effects. They concluded that the GSLT valid in all cases for small red shift values. In another study [87], the same authors investigated its validity in Brans-Dicke theory by introducing power law and logarithmic corrected entropy relations.

In the present paper, we will focus on the validity of GSLT at Hubble horizon in both equilibrium and non-equilibrium perspectives using a higher-order torsion derivatives based modified gravity. In the coming section, we will present some basic notions of this theory and the assumptions used for this work. Section 3 formulates the possible forms of first as well as GSL of thermodynamics and discuss the existence of its resulting non-equilibrium picture. For this purpose, we will consider two viable forms of $F$ function and some interesting cases of scale factor. We also investigate its validity using logarithmic corrected entropy there. In Sect. 4, we investigate the existence of equilibrium thermodynamics picture and check the validity of GSLT using same cases of function $F$ as well as the scale factor. Last section will summarize the whole discussion by highlighting the major results. 


\section{Basic formulation of $F\left(T,(\nabla T)^{2}, \square T\right)$ gravitational theory}

In this section, we will briefly present some basic formulation of the modified teleparallel theory under consideration. Here we will also specify the respective field equations along with the assumptions taken for this work. The relation of metric and vierbein $e_{A}^{\mu}$, the dynamical field of teleparallel gravity, is given by

$g_{\mu \nu}=\eta_{A B} e_{\mu}^{A} e_{v}^{B}$.

The torsion tensor describing the gravitational field in terms of Weitzenböck connection $\left(\Gamma_{v \mu}^{\lambda} \equiv e_{A}^{\lambda} \partial_{\mu} e_{v}^{A}\right)$ is expressed as

$T_{\mu \nu}^{\rho}=e_{A}^{\rho}\left(\partial_{\mu} e_{\nu}^{A}-\partial_{\nu} e_{\mu}^{A}\right)$.

The Lagrangian densities of teleparallel theory and its modified versions are based on the torsion scalar obtained by the contractions of the torsion tensor (2) as follows

$T \equiv \frac{1}{4} T^{\rho \mu \nu} T_{\rho \mu \nu}+\frac{1}{2} T^{\rho \mu \nu} T_{\nu \mu \rho}-T_{\rho \mu}{ }^{\rho} T_{\nu}^{\nu \mu}$.

The generalization of torsion based theories obtained by including higher-order derivative terms like $(\nabla T)^{2}$ and $\square T$ can be expressed by the following action [60]:

$\mathcal{A}=\frac{1}{2 \kappa^{2}} \int d x^{4} e F\left(T,(\nabla T)^{2}, \square T\right)+S_{m}\left(e_{\rho}^{A}, \psi_{m}\right)$,

where $S_{m}\left(e_{\rho}^{A}, \psi_{m}\right)$ denotes the ordinary matter part of action. Here $\kappa^{2}=8 \pi G$ and $F$ is a generic function of torsion scalar and its higher-order derivatives. Also, $e=\operatorname{det}\left(e_{\mu}^{A}\right)=\sqrt{-g}$. Further, these higher-order derivatives can be calculated by the formulas as

$$
\begin{gathered}
(\nabla T)^{2}=\eta^{A B} e_{A}^{\mu} e_{B}^{\nu} \nabla_{\mu} T \nabla_{\nu} T=g^{\mu \nu} \nabla_{\mu} T \nabla_{\nu} T, \\
\square T=\eta^{A B} e_{A}^{\mu} e_{B}^{\nu} \nabla_{\mu} \nabla_{\nu} T=g^{\mu \nu} \nabla_{\mu} \nabla_{\nu} T .
\end{gathered}
$$

For the sake of simplicity in calculations, we introduce the notations for higher-order derivatives as: $X_{1}=(\nabla T)^{2}$ and $X_{2}=\square T$. It is worthwhile to mention here that the action of simple $f(T)$ gravity can be recovered by removing the higher-order derivative terms, i.e., $X_{1}=X_{2}=0$. In terms of these new notations, the respective field equations obtained by the variation of the action (4) with respect to vierbein can be written as

$$
\begin{gathered}
\frac{1}{e} \partial_{\mu}\left(e F_{T} e_{A}{ }^{\tau} S_{\tau}{ }^{\rho \mu}\right)-F_{T} e_{A}{ }^{\tau} S_{\nu}{ }^{\mu \rho} T^{\nu}{ }_{\mu \tau} \\
+\frac{1}{4} e_{A}{ }^{\rho} F+\frac{1}{4} \sum_{i=1}^{2}\left\{F_{X_{i}} \frac{\partial X_{i}}{\partial e^{A}{ }_{\rho}}\right.
\end{gathered}
$$

$$
\begin{aligned}
& \left.-\frac{1}{e}\left[\partial_{\mu}\left(e F_{X_{i}} \frac{\partial X_{i}}{\partial \partial_{\mu} e^{A}{ }_{\rho}}\right)-\partial_{\mu} \partial_{\nu}\left(e F_{X_{i}} \frac{\partial X_{i}}{\partial \partial_{\mu} \partial_{\nu} e^{A}{ }_{\rho}}\right)\right]\right\} \\
& -\frac{1}{4 e} \partial_{\lambda} \partial_{\mu} \partial_{\nu}\left(e F_{X_{2}} \frac{\partial X_{2}}{\partial_{\lambda} \partial_{\mu} \partial_{\nu} e^{A}{ }_{\rho}}\right)=\frac{1}{2} e_{A}{ }^{\tau} \mathcal{T}^{(m)}{ }_{\tau}{ }^{\rho} .
\end{aligned}
$$

Here we have used the term "superpotential" expressed in terms of contortion tensor $K_{\rho}^{\mu \nu} \equiv-\frac{1}{2}\left(T^{\mu \nu}{ }_{\rho}-T_{\rho}^{\nu \mu}-\right.$ $T_{\rho}^{\mu \nu}$ ) and is defined by the following relations:

$S_{\rho}^{\mu \nu} \equiv \frac{1}{2}\left(K_{\rho}^{\mu \nu}+\delta_{\rho}^{\mu} T_{\theta}^{\theta v}-\delta_{\rho}^{\nu} T_{\theta}^{\theta \mu}\right)$.

Further, the notations $F_{T}$ and $F_{X i} ;(i=1,2)$ stand for the derivatives of the generic function $F$ with respect to the subscript variable, i.e., $\frac{\partial F}{\partial T}, \frac{\partial F}{\partial X_{i}}$, respectively. Also, the contribution of ordinary matter given on left side of (7) can be defined as follows

$e_{A}{ }^{\tau} \mathcal{T}^{(m)}{ }_{\tau}^{\rho} \equiv-\frac{1}{e} \frac{\delta S_{m}}{\delta e^{A}{ }_{\rho}}$.

Consider the spatially flat FRW universe geometry with cosmic radius $a(t)$ given by the line element

$d s^{2}=d t^{2}-a^{2}(t)\left(d x^{2}+d y^{2}+d z^{2}\right)$.

The corresponding set of vierbein components are

$e_{\mu}^{A}=\operatorname{diag}(1, a(t), a(t), a(t))$.

Here the energy-momentum tensor of ordinary matter source is assumed to be perfect fluid given by

$T_{\mu \nu}=\left(\rho_{m}+p_{m}\right) u_{\mu} u_{\nu}-p_{m} g_{\mu \nu}$,

where $\rho_{m}$ and $p_{m}$ represent the density and pressure of ordinary matter, respectively. Under these assumptions, the field equations finally take the following form:

$$
\begin{aligned}
& F_{T} H^{2}+\left(24 H^{2} F_{X_{1}}+F_{X_{2}}\right)(3 H \dot{H}+\ddot{H}) H \\
& \quad+F_{X_{2}} \dot{H}^{2}+\left(3 H^{2}-\dot{H}\right) H \dot{F}_{X_{2}} \\
& \quad+24 H^{3} \dot{H} \dot{F}_{X_{1}}+H^{2} \ddot{F}_{X_{2}}+\frac{F}{12}=\frac{\rho_{m}}{6}, \\
& F_{T} \dot{H}+H \dot{F}_{T}+24 H\left[2 H \ddot{H}+3\left(\dot{H}+H^{2}\right) \dot{H}\right] \dot{F}_{X_{1}} \\
& \quad+12 H \dot{H} \dot{F}_{X_{2}}+24 H^{2} \dot{H} \ddot{F}_{X_{1}} \\
& \quad+\left(\dot{H}+3 H^{2}\right) \ddot{F}_{X_{2}}+24 H^{2} F_{X_{1}} \dddot{H}+H \dddot{F}_{X_{2}} \\
& \quad+24 F_{X_{1}} \dot{H}^{2}\left(12 H^{2}+\dot{H}\right) \\
& +24 H F_{X_{1}}\left(4 \dot{H}+3 H^{2}\right) \ddot{H}=-\frac{p_{m}}{2},
\end{aligned}
$$

where $H=\dot{a} / a$ represents the Hubble parameter and the dot denotes the cosmic time rate of change. Equations (9) and (10) can be rearranged to following forms:

$3 H^{2}=\kappa_{e f f}^{2} \rho_{e f f}, \quad \dot{H}=-\kappa^{2}$ eff $\left(\rho_{e f f}+p_{e f f}\right)$,

where the effective energy density and pressure are the combinations $\rho_{e f f}=\rho_{m}+\rho_{T}$ and $p_{e f f}=p_{m}+p_{T}$, respectively. 
Also, the effective coupling is defined as $\kappa^{2}$ eff $=\frac{\kappa^{2}}{2 F_{T}}$. These contributions of density and pressure due to torsion are given by

$$
\begin{aligned}
\rho_{T}= & \frac{1}{\kappa^{2}}\left[-6\left(24 H^{2} F_{X_{1}}+F_{X_{2}}\right)(3 H \dot{H}+\ddot{H}) H\right) \\
& -6 F_{X_{2}} \dot{H}^{2}-6\left(3 H^{2}-\dot{H}\right) H \dot{F}_{X_{2}} \\
& \left.-144 H^{3} \dot{H}_{X_{1}}-6 H^{2} \ddot{F}_{X_{2}}-\frac{F}{2}\right], \\
p_{T}= & \frac{2}{\kappa^{2}}\left[H \dot{F}_{T}+24 H\left[2 H \ddot{H}+3\left(\dot{H}+H^{2}\right) \dot{H}\right] \dot{F}_{X_{1}}\right. \\
& +12 H \dot{H} \dot{F}_{X_{2}}+24 H^{2} \dot{H} \ddot{F}_{X_{1}} \\
& +\left(\dot{H}+3 H^{2}\right) \ddot{F}_{X_{2}}+24 H^{2} F_{X_{1}} \dddot{H}+H \dddot{F}_{X_{2}} \\
& +24 F_{X_{1}} \dot{H}^{2}\left(12 H^{2}+\dot{H}\right) \\
& \left.+24 H F_{X_{1}}\left(4 \dot{H}+3 H^{2}\right) \ddot{H}-3 H^{2} F_{T}\right] .
\end{aligned}
$$

For this spatially flat geometry, the torsion scalar and its derivatives $(\nabla T)^{2}$ and $\square T$ turn out to be

$$
\begin{aligned}
T & =-6 H^{2}, \quad X_{1}=144 H^{2} \dot{H}^{2}, \\
X_{2} & =-12\left[\dot{H}\left(\dot{H}+3 H^{2}\right)+H \ddot{H}\right] .
\end{aligned}
$$

Also, the ordinary matter satisfies the usual continuity equation and is given by

$\dot{\rho}_{m}+3 H\left(\rho_{m}+p_{m}\right)=0$.

It is worthwhile to mention here that similarly, the effective density and pressure satisfies the continuity equation

$\dot{\rho}_{e f f}+3 H\left(\rho_{e f f}+p_{e f f}\right)=0$,

which consequently gives rise to the non-conservation of its torsion scalar counterparts due to the presence of an extra term on left side as follows

$\dot{\rho}_{T}+3 H\left(\rho_{T}+p_{T}\right)=\frac{T}{\kappa^{2}} \dot{F}_{T}$

By assuming the barotropic equation of state $p_{m}=\omega_{m} \rho_{m}$; $0 \leq \omega_{m} \leq 1$, the integration of the continuity equation leads to the following relation

$\rho_{m}=\rho_{m 0} a^{-3\left(1+\omega_{m}\right)}$,

where $\rho_{m 0}$ represents an arbitrary constant of integration.

\section{Non-equilibrium and equilibrium perspectives of thermodynamics in $F\left(T, X_{1}, X_{2}\right)$ gravity}

In this section, we present a brief discussion on the first and generalized second law of thermodynamics by considering the perspective of non-equilibrium. It has already been discussed in literature $[80,88,89]$ that such picture exits in the extended gravitational theories based on curvature or torsion matter couplings like $f(R, T), f(R, T, Q), f\left(T, L_{m}\right)$ and $f\left(R, L_{m}\right)$ theories.

\subsection{First law of thermodynamics}

Here we describe the possible form of first thermodynamical law in this modified gravity and investigate the issue of nonequilibrium picture there. For a flat FRW geometry, the radius of dynamical apparent horizon in terms of $h^{\alpha \beta}$ given by the condition $h^{\alpha \beta} \partial_{\alpha} \tilde{R}_{A} \partial_{\beta} \tilde{r}_{A}=0$, takes the form

$\tilde{R}_{A}=\frac{1}{H}$

Its time rate of change yields the following equation:

$\frac{d \tilde{R}_{A}}{d t}=\tilde{R}^{3} H \kappa_{e f f}^{2}\left(\rho_{e f f}+p_{e f f}\right)$.

After simplifying, the above equation can be written as

$\frac{F_{T} d \tilde{R}_{A}}{G}=4 \pi \tilde{R}_{A}^{3} H\left(\rho_{e f f}+p_{e f f}\right) d t$.

The area of the horizon is defined as $A=4 \pi \tilde{R}_{A}^{2}$ and the temperature associated with this horizon in terms of surface gravity $\kappa_{s g}$ is defined by $T_{A}=\kappa_{s g} / 2 \pi$, where

$\kappa_{s g}=\frac{1}{2 \sqrt{-h}} \partial_{\alpha}\left(\sqrt{-h} h^{\alpha \beta} \partial_{\beta} \tilde{R}_{A}\right)$.

For flat FRW model, it will take the form

$-\frac{1}{\tilde{R}_{A}}\left(1-\frac{\dot{\tilde{R}}_{A}}{2 H \tilde{R}_{A}}\right)=-\frac{\tilde{R}_{A}}{2}\left(2 H^{2}+\dot{H}\right)$.

On multiplication by the factor $\left(1-\frac{\dot{\tilde{R}}_{A}}{2 H \tilde{R}_{A}}\right)=-2 \pi \tilde{R} T_{A}$, the above equation leads to the following relation:

$$
\begin{aligned}
T_{A} d\left(\frac{A F_{T}}{4 G}\right)= & -\left(4 \pi \tilde{R}_{A}^{3} H d t-2 \pi \tilde{R}_{A}^{2} \dot{\tilde{R}}_{A}\right)\left(\rho_{e f f}+p_{e f f}\right) \\
& +\frac{\pi \tilde{R}_{A}^{2}}{G} T_{A} d F_{T} .
\end{aligned}
$$

The Bekenstein-Hawking entropy relation [63-65] suggests $S=A / 4 G$. Like many other modified gravity theories (for example, [80,86,88,89]), this relation is modified by the inclusion of $G_{e f f}$ instead of $G$. Consequently, in this theory, it takes the form $S_{A}=\frac{A F_{T}}{4 G}$. Thus the last equation can be re-written as 


$$
\begin{aligned}
T_{A} d \tilde{S}_{A}= & \left(2 \pi \tilde{R}_{A}^{2} \dot{\tilde{R}}_{A}-4 \pi \tilde{R}_{A}^{3} H d t\right)\left(\rho_{e f f}+p_{e f f}\right) \\
& +\frac{\pi \tilde{R}_{A}^{2}}{G} T_{A} d F_{T} .
\end{aligned}
$$

The Misner-Sharp energy defined by the relation $E=\frac{\tilde{R}_{A}}{2 G_{\text {eff }}}$ or equivalently, $E=\rho_{e f f} V$ provides the total matter energy density of universe (a sphere of radius $\tilde{R}_{A}$ at the apparent horizon). Here the volume of the universe is given by the equation $V=4 / 3 \pi \tilde{R}_{A}^{3}$. In this modified teleparallel gravity, this relation leads to

$$
\begin{aligned}
d E= & 4 \pi \tilde{R}_{A}^{2}\left(\rho_{m}+\rho_{T}\right) d \tilde{R}_{A}-4 \pi \tilde{R}_{A}^{3} H\left(\rho_{e f f}+p_{e f f}\right) d t \\
& +\frac{\tilde{R}_{A}^{3}}{2 G}\left(d F_{T}\right) .
\end{aligned}
$$

Inserting this $d E$ in Eq. (20), we obtain

$$
\begin{aligned}
T_{A} d \tilde{S}_{A}= & d E+2 \pi \tilde{r}_{A}^{2}\left(p_{e f f}-\rho_{e f f}\right) d \tilde{R}_{A} \\
& +\frac{\tilde{R}_{A}}{G}\left(3+\pi \tilde{R}_{A} T_{A}\right) d F_{T} .
\end{aligned}
$$

Also, the total work density is defined by the equation [85]

$W=-\frac{1}{2}\left(T^{(m) \alpha \beta} h_{\alpha \beta}+\tilde{T}^{(d e) \alpha \beta} h_{\alpha \beta}\right)=\frac{1}{2}\left(\rho_{e f f}-p_{e f f}\right)$.

where the notations $T^{(m) \alpha \beta}$ and $\tilde{T}^{(d e)}$ stand for the energy densities due to ordinary and dark matter, respectively. Introducing work density in Eq. (21) leads to the final form of first law of thermodynamics given by

$$
T_{A} d \tilde{S}_{A}+T_{A} d \tilde{S}_{p}=d E-W d V
$$

where the term $d \tilde{S}_{p}=\frac{\tilde{R}_{A}}{G T_{A}}\left(3+\pi \tilde{R}_{A} T_{A}\right) d F_{T}$ is due to the entropy production in non-equilibrium thermodynamics. Thus, we conclude that in this extended teleparallel gravity, the form of first law of thermodynamics is modified by the presence of a surplus term. This in agreement with the already available results in literature for $f(R), f(R, T), f(R, T, Q)$ theories as well as generalized Gauss-Bonnet gravity where a surplus term exist giving rise to non-equilibrium thermodynamics there.

\subsection{GSLT in modified $f(T)$ gravity}

In the present section, we explore the issue of GSLT validity in the context of this generalized teleparallel gravity. The GSLT suggests that function obtained by the sum of entropies of horizon and ordinary matter fluid components always increases versus cosmic time. This issue has been already investigated in the context of various modified theories like $f(R), f(R)$ theory involving matter geometry coupling, $f(T), f(R, T), f(R, T, Q), f\left(R, L_{m}\right)$ and scalar- tensor theories. Here we will utilize new form of first law of thermodynamics obtained in the previous section. Mathematically, GSLT can be written as

$\dot{\tilde{S}}_{\text {total }}=\dot{\tilde{S}}_{h}+\dot{\tilde{S}}_{p}+\dot{\tilde{S}}_{i n} \geq 0$

where the notations $\tilde{S}_{h}, \tilde{S}_{p}$ and $\tilde{S}_{i n}$ stand for horizon entropy, entropy production term and entropy of matter components inside horizon, respectively. First law of thermodynamics (23) provides the relation:

$T_{i} d \tilde{S}_{i}=d E_{i}+p_{i} d V-T_{i} d \tilde{S}_{p}$,

which can also be written as

$T_{i n} \dot{\tilde{S}}_{i n}=\left(\rho_{i}+p_{i}\right) 4 \pi \tilde{R}_{A}^{2}\left(\dot{\tilde{R}}_{A}-H \tilde{R}_{A}\right)+\frac{4}{3} \pi \tilde{R}_{A}^{3} Q_{i}-T_{i n} \dot{\tilde{S}}_{p}$,

where $T_{\text {in }}$ denotes the temperature for all components inside the horizon, $Q_{i}$ represents the ith term interaction component. Taking summation of all inside horizon components entropies, we get

$\sum Q_{i}=0, \quad \sum\left(\rho_{i}+p_{i}\right)=\rho_{e f f}+p_{e f f}$.

Consequently, we have

$T_{i n} \dot{\tilde{S}}_{i n}=\left(\rho_{e f f}+p_{e f f}\right) 4 \pi \tilde{R}_{A}^{2}\left(\dot{\tilde{R}}_{A}-H \tilde{R}_{A}\right)-T_{i n} \dot{\tilde{S}}_{p}$

Further, after an easy calculation, one can write the last equation as follows:

$\dot{\tilde{S}}_{i n}+\dot{\tilde{S}}_{p}=\frac{4 \pi}{G} \frac{\dot{H}\left(\dot{H}+H^{2}\right) F_{T}}{\left(2 H^{2}+\dot{H}\right) H^{3}}$.

Also, from the Bekenstein-Hawking entropy relation, one can find

$\dot{\tilde{S}}_{h}=\frac{\pi}{G H^{2}}\left(\dot{F}_{T}-2 \frac{\dot{H}}{H} F_{T}\right)$

Thus, from Eqs. (24), (25) and (26), the GSLT constraint takes the following form

$\dot{\tilde{S}}_{t o t}=\frac{4 \pi}{G}\left(\frac{\dot{H}\left(\dot{H}+H^{2}\right) F_{T}}{\left(2 H^{2}+\dot{H}\right) H^{3}}+\frac{1}{4 H^{2}}\left\{\dot{F}_{T}-2 \frac{\dot{H}}{H} F_{T}\right\}\right) \geq 0$

In the upcoming subsections, we will explore the validity of this constraint using two different functional forms of $F$ and in last, by considering the logarithmic entropy correction term. 


\subsubsection{The validity of GSLT constraint for a function independent of $X_{2}$}

Here we will explore the validity of GSLT using the form of $F$ that is independent of $X_{2}$ given as follows

$F\left(T, X_{1}, X_{2}\right)=T+\frac{\alpha_{1} X_{1}}{T^{2}}+\alpha_{2} e^{\frac{\delta X_{1}}{T^{4}}}$,

where $\alpha_{1}, \alpha_{2}$ and $\delta$ are all dimensionless constants. This form of $F$ has already been used in literature for checking the validity of energy constraints as well as the stability using fixed point theory $[60,90]$. The GSLT constraint for this functional form is given by

$$
\begin{aligned}
\dot{\tilde{S}}_{t o t}= & \frac{4 \pi}{G}\left[\frac{\dot{H}^{2}+\dot{H} H^{2}}{H^{3}\left(2 H^{2}+\dot{H}\right)}\left(1-\frac{2 \alpha_{1} X_{1}}{T^{3}}-\frac{4 \alpha_{2} \delta X_{1}}{T^{5}} e^{\frac{\delta X_{1}}{T^{4}}}\right)\right. \\
& +\frac{1}{4 H^{2}}\left[\left\{\left(\frac{6 \alpha_{1} X_{1}}{T^{4}}+\left(\frac{16 \alpha_{2} \delta^{2} X_{1}^{2}}{T^{10}}+\frac{20 \alpha_{2} \delta_{1}}{T^{6}}\right) e^{\frac{\delta X_{1}}{T^{4}}}\right) \dot{T}\right.\right. \\
& \left.+\left(-\frac{2 \alpha_{1}}{T^{3}}-\frac{4 \alpha_{2} \delta}{T^{5}}\left(1+\frac{\delta}{T^{4}}\right) e^{\frac{\delta X_{1}}{T^{4}}}\right) \dot{X}_{1}\right\} \\
& \left.\left.-\frac{2 \dot{H}}{H}\left\{1-\frac{2 \alpha_{1} X_{1}}{T^{3}}-\frac{4 \alpha_{2} \delta X_{1}}{T^{5}} e^{\frac{\delta X_{1}}{T^{4}}}\right\}\right]\right] \geq 0 .
\end{aligned}
$$

Now we will discuss the validity of GSLT constraint (29) by taking different possibilities of scale factor given as follows

- Constant Hubble parameter: $H=H_{0}$, where $H_{0}$ is recent value of Hubble parameter, i.e., the de Sitter model.

- Expressing the higher order time rates in terms of cosmographic parameters like $q, r, s$ etc.

- Power law form: $a(t)=a_{0}\left(t_{s}-t\right)^{-b}$, where $a_{0}$ is the present value of the scale factor and $t_{s} \geq t, b>0$.

- Intermediate form: $a(t)=e^{b_{1} t^{\beta}}$, where $b_{1}$ is any positive constant and $0<\beta<1$.

In the first place, we evaluate the GSLT for the choice of de-Sitter model having constant Hubble parameter $H=H_{0}$. In this case, it is found that GSLT is trivially satisfied as all the derivatives vanish for this choice.

Secondly, we discuss the validity of GSLT constraint by introducing the cosmographic parameters. Here we define some interesting cosmographic parameters depending on higher-order derivatives of scale factor obtained by the Taylor's series expansion of scale factor like deceleration, jerk, snap and lerk parameters etc.. These parameters are defined as follows

$$
\begin{aligned}
& q=-\frac{1}{H^{2}} \frac{a^{(2)}}{a}, \quad j=\frac{1}{H^{3}} \frac{a^{(3)}}{a}, \\
& s=\frac{1}{H^{4}} \frac{a^{(4)}}{a}, \quad l=\frac{1}{H^{5}} \frac{a^{(5)}}{a} .
\end{aligned}
$$

It is worthwhile to mention here that all the higher-order derivatives of Hubble parameter can be expressed as a linear combination of these cosmographic parameters. For example, first four order time rates of Hubble parameter in terms of these cosmographic parameters can be written as

$$
\begin{aligned}
\dot{H} & =-H^{2}(1+q), \quad \ddot{H}=H^{3}(j+3 q+2), \\
\dddot{H} & =H^{4}(s-2 j-5 q-3), \\
H^{(4)} & =H^{5}(l-5 s+10(q+2) j+30(q+2) q+24) .
\end{aligned}
$$

Consequently, the terms like torsion scalar, $X_{1}$ and $X_{2}$ and their corresponding time rates can be expressed in terms of these cosmographic parameters as given below

$$
\begin{aligned}
T= & -6 H^{2}, \quad X_{1}=144 H^{6}(1+q)^{2}, \\
X_{2}= & -12\left(H^{4}(1+q)^{2}-3 H^{4}(1+q)+H^{4}(j+3 q+2)\right), \\
\dot{T}= & 12 H^{3}(1+q), \\
\dot{X}_{1}= & -288 H^{7}(1+q)\left\{(1+q)^{2}+j+3 q+2\right\}, \\
\dot{X}_{2}= & 12 H^{5}\{3(1+q)(j+3 q+2)-3(j+3 q+2) \\
& \left.-6(1+q)^{2}(s-2 j-5 q-3)\right\} .
\end{aligned}
$$

In this case, for the graphical analysis, we consider the present values of these cosmographic quantities as suggested in literature [91] and are given by $H_{0}=0.718, q_{0}=-0.64, j_{0}=$ $1.02, s_{0}=-0.39$ and $l_{0}=4.05$. For these values, the quantities of Eq. (31) become

$T=-3.0931, \quad X_{1}=2.5569, \quad X_{2}=-0.4771$,

$\dot{T}=1.5590, \quad \dot{X}_{1}=-12.5409, \quad \dot{X}_{2}=-0.8654$.

By using these values, we explored the possible ranges of model parameters namely $\alpha_{1}, \alpha_{2}$ and $\delta$ using region graph as given in Fig. 1. The detailed possible ranges of these parameters for which GSLT condition remain valid are given in Table 1.

Now we consider the possibility of power law form of expansion factor given by the relation $a(t)=a_{0}\left(t_{s}-\right.$

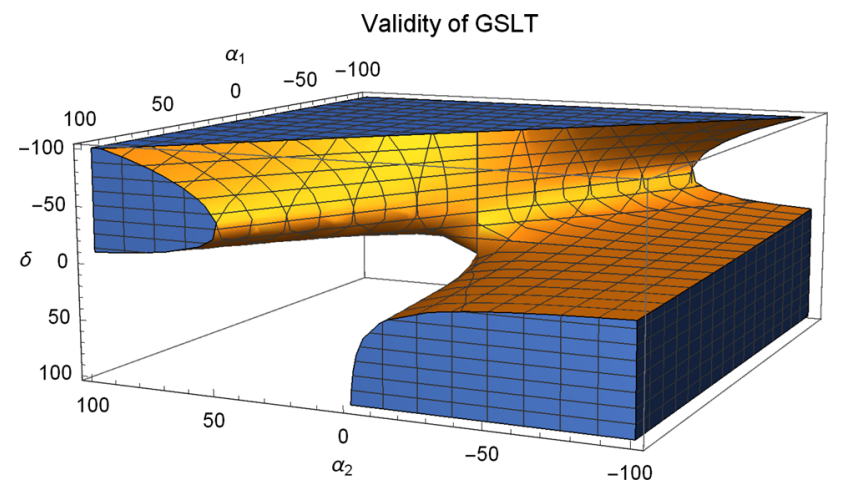

Fig. 1 The plot represents the validity regions for GSLT constraint in terms of cosmographic parameters for model (28) 
Table 1 Validity regions of $\dot{\tilde{S}}_{t o t} \geq 0$ for different models

\begin{tabular}{llr}
\hline$F\left(T, X_{1}, X_{2}\right)$ models & Validity of GSLT & $\begin{array}{l}\text { Cosmographic parameters } \\
q, j, r, s, l \text { parameters }\end{array}$ \\
\hline Model 1 & Non-equilibrium picture & $\begin{array}{l}\text { if } \delta \geq 20 \& \alpha_{2} \leq-20, \forall \alpha_{1} \\
\text { if } \delta \leq 0 \& \alpha_{2} \geq 0 \text { with } \alpha_{1} \leq 0\end{array}$ \\
& & Logarithmic corrected entropy \\
$F\left(T, X_{1}, X_{2}\right)=T+\frac{\alpha_{1} X_{1}}{T^{2}}+\alpha_{2} e^{\frac{\delta X_{1}}{T^{4}}}$ & & if $\delta \geq 15 \& \alpha_{2} \leq-30, \forall \alpha_{1}$ \\
& Equilibrium picture & if $\delta \leq-20 \& \alpha_{2} \geq 85, \forall \alpha_{1}$ \\
& & if $\delta \geq 40 \& \alpha_{2} \leq-10, \forall \alpha_{1}$ \\
& if $\delta \leq-15 \& \alpha_{2} \leq-20, \forall \alpha_{1}$ \\
& Non-equilibrium picture & if $\beta_{3} \geq 70 \forall \beta_{1} \& \beta_{2}$ \\
Model 2 & Logarithmic corrected entropy & if $\beta \leq-35 \& \beta_{3} \geq 50 \forall \beta_{2}$ \\
$F\left(T, X_{1}, X_{2}\right)=T+\frac{\beta_{1} X_{2}}{T}+\frac{\beta_{2} X_{2}^{2}}{T^{3}}+\beta_{3} e^{\frac{\sigma X_{2}}{T^{3}}}$ & Equilibrium picture & if $\beta_{2} \leq 50, \forall \beta_{1} \& \beta_{3}$
\end{tabular}

$t)^{-b}, b>0, t_{s} \geq t$. Here the point $t=t_{s}$ leads to the presence of a Big Rip singularity in this super accelerated cosmos model $[92,93]$. In this case, the Hubble parameter, Torsion scalar and the terms $X_{1}, X_{2}$ along with their derivative terms turn out to be as follows

$$
\begin{aligned}
H & =\frac{b}{t_{s}-t}, \quad T=-\frac{6 b^{2}}{\left(t_{s}-t\right)^{2}}, \\
\dot{H} & =\frac{b}{\left(t_{s}-t\right)^{2}}, \quad \dot{T}=-\frac{12 b^{2}}{\left(t_{s}-t\right)^{3}}, \\
X_{1} & =\frac{144 b^{4}}{\left(t_{s}-t\right)^{6}}, \quad \dot{X}_{1}=\frac{864 b^{4}}{\left(t_{s}-t\right)^{7}}, \\
X_{2} & =-\frac{36 b^{2}(1+b)}{\left(t_{s}-t\right)^{4}}, \quad \dot{X}_{2}=-\frac{144 b^{2}(1+b)}{\left(t_{s}-t\right)^{5}} .
\end{aligned}
$$

Here $b$ is the power law parameter and $\alpha_{1}, \alpha_{2}, \delta$ are the model parameters. In this discussion, we fix $\delta$ and evaluate the validity ranges for $\alpha_{1}$ and $\alpha_{2}$. If $\delta>0$ then validity of GSLT requires $\alpha_{2} \geq 0$ for all values of $\alpha_{1}$ whereas if $\delta \leq 0$ then GSLT is satisfied for $\alpha_{1} \geq 0$ with all values of $\alpha_{2}$ as shown in Table 2. The graphical illustration of validity of GSLT constraint is shown in Fig. 2 for some particular cases. In some cases, it is not obvious to find the exact region of validity, one of such cases is shown in right plot of Fig. 2. In left of Fig. 2, we have selected one particular validity range and presented its evolution of GSLT for different values of $\delta$.

Now we will discuss the validity of the GSLT constraint using the intermediate form of expansion radius given by $a(t)=e^{b_{1} t^{\beta}}, 0<\beta<1, b>0$. Such form of expansion factor is very significant as it plays an important role in the description of inflationary scenario and hence compatible with the astrophysical evidences [94]. For this form of expansion radius, the cosmological parameters like Hubble parameter, torsion scalar, terms $X_{1}, X_{2}$ and its first order time rates take the following form

$$
H=b_{1} \beta t^{\beta-1}, \quad \dot{H}=b_{1} \beta(\beta-1) t^{\beta-2},
$$

$$
\begin{aligned}
T= & -6 b_{1}^{2} \beta^{2} t^{2(\beta-1)}, \quad \dot{T}=-12 b_{1}^{2} \beta^{2}(\beta-1) t^{2 \beta-3}, \\
X_{1}= & 144 b_{1}^{4} \beta^{4}(\beta-1)^{2} t^{4 \beta-6}, \\
\dot{X}_{1}= & 288 b_{1}^{4} \beta^{4}(\beta-1)^{2}(2 \beta-3) t^{4 \beta-7}, \\
X_{2}= & -12\left\{b_{1}^{2} \beta^{2}(\beta-1)(2 \beta-3) t^{2 \beta-4}\right. \\
& \left.+3 b_{1}^{3} \beta^{3}(\beta-1) t^{3 \beta-4}\right\}, \\
\dot{X}_{2}= & -12\left\{b_{1}^{2} \beta^{2}(\beta-1)(2 \beta-3)(2 \beta-4) t^{2 \beta-5}\right. \\
& \left.+3 b_{1}^{3} \beta^{3}(\beta-1)(3 \beta-4) t^{3 \beta-5}\right\} .
\end{aligned}
$$

In intermediate form, we have two additional parameters $b$ and $\beta$ along with the model parameters $\alpha_{1}, \alpha_{2}$ and $\delta$. Herein, we set $b_{1}=2, \sigma=2$ and $\beta=0.5$. We fix the parameter $\delta$ to explore the validity of GSLT depending on the parameters $\alpha_{1}$ and $\alpha_{2}$. If $\delta>0$, then validity of GSLT requires $\alpha_{1} \geq 0$ and $\alpha_{2} \leq-20$ whereas if $\delta<0$ it requires $\alpha_{1} \geq 0$ and $\alpha_{2} \leq-5$. Moreover, in case of $\delta=0$, one need to set $\alpha_{1} \leq 0$ along with all values $\alpha_{2}$.

In left plot of Fig. 3, we represent one particular region of validity whereas in the right plot, we use the validity range of parameters $\alpha_{1}$ and $\alpha_{2}$ to show the evolution of GSLT versus $\delta$.

\subsubsection{The validity of GSLT constraint for a function independent of $X_{1}$}

Now we will consider the form of generic function $F\left(T, X_{1}, X_{2}\right)$ independent of the term $X_{1}$ which is defined by the following relation

$F\left(T, X_{1}, X_{2}\right)=T+\frac{\beta_{1} X_{2}}{T}+\frac{\beta_{2} X_{2}^{2}}{T^{3}}+\beta_{3} e^{\frac{\sigma X_{2}}{T^{3}}}$,

where $\beta_{i} ; i=1,2,3$ and $\sigma$ are all arbitrary constant parameters. In this case, the GSLT constraint will take the form 
Table 2 Validity regions of $\dot{\tilde{S}}_{t o t} \geq 0$ for different models

\begin{tabular}{|c|c|c|c|c|}
\hline \multirow{2}{*}{$\begin{array}{l}F\left(T, X_{1}, X_{2}\right) \text { Mod- } \\
\text { els }\end{array}$} & \multirow[t]{2}{*}{ Validity of GSLT } & \multicolumn{3}{|l|}{ Various scale factors } \\
\hline & & $\begin{array}{l}\text { de-Sitter model } \\
H=H_{0}\end{array}$ & $\begin{array}{l}\text { Power law form } \\
a(t)=a_{0}\left(t_{s}-t\right)^{-b}\end{array}$ & $\begin{array}{l}\text { intermediate form } \\
a(t)=e^{b_{1} t^{\beta}}\end{array}$ \\
\hline \multirow[t]{2}{*}{ Model 1} & $\begin{array}{l}\text { Non-equilibrium } \\
\text { picture }\end{array}$ & Trivially satisfied & if $\delta>0 ; \alpha_{2} \geq 0 \& \forall \alpha_{1}$ & if $\delta>0 ; \alpha_{1} \geq 0 \& \alpha_{2} \leq-20$ \\
\hline & & & $\begin{array}{l}\text { if } \delta=0, \alpha_{1} \geq 0 \& \forall \alpha_{2} \\
\text { if } \delta<0, \alpha_{1} \geq 0 \& \forall \alpha_{2}\end{array}$ & $\begin{array}{l}\text { if } \delta=0, \alpha_{1} \leq 0 \& \forall \alpha_{2} \\
\text { if } \delta<0 ; \alpha_{1} \geq 0 \& \alpha_{2} \leq-5\end{array}$ \\
\hline \multirow[t]{3}{*}{$\begin{array}{l}F\left(T, \quad X_{1}, \quad X_{2}\right)= \\
T+\frac{\alpha_{1} X_{1}}{T^{2}}+\alpha_{2} e^{\frac{\delta X_{1}}{T^{4}}}\end{array}$} & $\begin{array}{l}\text { Logarithmic } \\
\text { corrected entropy }\end{array}$ & $\lambda_{1}=-6.06+0.16 \lambda_{2}, \forall \lambda_{2}$ & if $\delta>0 ; \alpha_{2} \geq 10 \& \forall \alpha_{1}$ & if $\delta>0 ; \alpha_{1} \leq 0 \& \alpha_{2} \leq-11$ \\
\hline & & & $\begin{array}{l}\text { if } \delta=0 ; \alpha_{1} \leq-18 \& \forall \alpha_{2} \\
\text { if } \delta<0 ; \alpha_{1} \leq 0 \& \forall \alpha_{2}\end{array}$ & $\begin{array}{l}\text { if } \delta=0 ; \alpha_{1} \leq-30 \& \forall \alpha_{2} \\
\text { if } \delta<0 ;\left(\alpha_{1}, \alpha_{2}\right) \leq-15\end{array}$ \\
\hline & Equilibrium picture & $\alpha_{2}=3.11$ & $\begin{array}{l}\text { if } \delta>0 ; \alpha_{2} \geq 1 \& \forall \alpha_{1} \\
\text { if } \delta=0 ; \alpha_{2} \leq-1 \& \forall \alpha_{1} \\
\text { if } \delta<0 ; \alpha_{2} \leq-1 \& \forall \alpha_{1}\end{array}$ & $\begin{array}{l}\text { if } \delta>0 ;\left(\alpha_{1}, \alpha_{2}\right) \geq 0 \text {, Later times } \\
\text { if } \delta=0 ; \alpha_{1} \geq 0 \& \alpha_{2} \leq 0 \\
\text { if } \delta<0 ; \alpha_{1} \geq 5 \& \alpha_{2} \leq 0\end{array}$ \\
\hline \multirow[t]{2}{*}{ Mode 2} & $\begin{array}{l}\text { Non-equilibrium } \\
\text { picture }\end{array}$ & Trivially satisfied & if $\beta_{3}<0 ; \beta_{1} \& \beta_{2}$ & if $0<\beta_{3}<80 ; \forall\left(\beta_{1}, \beta_{2}\right)<0$ \\
\hline & & & if $\beta_{3}=0 ; \forall \beta_{1} \& \beta_{2} \leq-20$ & $\begin{array}{l}\text { if } \beta_{3} \geq 80 ; \forall \beta_{1} \& \beta_{2} \\
\text { if } \beta_{3}=0 ; \forall\left(\beta_{1}, \beta_{2}\right)>0\end{array}$ \\
\hline \multirow{3}{*}{$\begin{array}{l}F\left(T, X_{1}, X_{2}\right)= \\
T+\frac{\beta_{1} X_{2}}{T}+\frac{\beta_{2} X_{2}^{2}}{T^{3}}+ \\
\beta_{3} e^{\frac{\sigma X_{2}}{T^{3}}}\end{array}$} & $\begin{array}{l}\text { Logarithmic } \\
\text { corrected entropy }\end{array}$ & Trivially satisfied & if $\beta_{3}=0 ; \beta_{1} \geq 0 \& \beta_{2} \leq-20$ & if $\beta_{3}>0 ; \beta_{1} \leq-80, \forall \beta_{2}$ \\
\hline & & & if $\beta_{3}<0 ; \forall \beta_{1} \& \beta_{2}$ & $\begin{array}{l}\text { if } \beta_{3}=0 ;\left(\beta_{1}, \beta_{2}\right) \geq 0 \\
\text { if } \beta_{3}<0 ; \forall \beta_{1} \& \beta_{2}\end{array}$ \\
\hline & Equilibrium picture & $\beta_{3}=-3.11$ & if $\beta_{3}>0, \forall \beta_{1} \& \beta_{2}$ & $\begin{array}{l}\text { if } \beta_{3}>0, \forall \beta_{1} \& \beta_{2} \text { Later times } \\
\text { if } \beta_{3}<0 \text {, Fig. (10) }\end{array}$ \\
\hline
\end{tabular}

$$
\begin{aligned}
\dot{\tilde{S}}_{t o t}= & \frac{4 \pi}{G}\left[\left\{\frac{\dot{H}\left(\dot{H}+H^{2}\right)}{\left(2 H^{2}+\dot{H}\right) H^{3}}-\frac{\dot{H}}{2 H^{3}}\right\}\right. \\
& \times\left\{1-\frac{\beta_{1} X_{2}}{T^{2}}-\frac{3 \beta_{2} X_{2}^{2}}{T^{4}}-\frac{3 \sigma \beta_{3} X_{2}}{T^{4}} e^{\frac{\sigma X_{2}}{T^{3}}}\right\} \\
& +\frac{1}{4 H^{2}}\left\{\left(\frac{2 \beta_{1} X_{2}}{T^{3}}+\frac{12 \beta_{2} X_{2}^{2}}{T^{5}}\right.\right. \\
& \left.+3 \sigma \beta_{3} X_{2}\left(\frac{4}{T^{5}}+\frac{3 \sigma X_{2}}{T^{4}}\right) e^{\frac{\sigma X_{2}}{T^{3}}}\right) \dot{T} \\
& \left.\left.+\left(-\frac{\beta_{1}}{T^{2}}-\frac{6 \beta_{2} X_{2}}{T^{4}}-\frac{3 \sigma \beta_{3}}{T^{4}}\left(1+\frac{\sigma}{T^{3}}\right) e^{\frac{\sigma X_{2}}{T^{3}}}\right) \dot{X}_{2}\right\}\right] \geq 0 .
\end{aligned}
$$

Using previously defined four different cases of expansion factor namely constant Hubble parameter, cosmographic parameters, power law and intermediate forms along with the corresponding relations of torsion scalar, $X_{1}$ and $X_{2}$ with their time rates given by Eqs. (31-33), we will check the compatibility of this GSLT condition and explore the possible choices of free model parameters. Here, we have four model parameters $\beta_{1}, \beta_{2}, \beta_{3}$ and $\sigma$. If $\beta_{2}=0$, then one can retrieve the results similar to the previous model. Herein, we set $b=1$ and $\sigma=2$. For de Sitter case, it is seen that the GSLT constraint trivially holds. For the case of cosmographic parameters, by using the previously defined present values of these parameters, the possible ranges of $\beta_{1}, \beta_{2}$ and $\beta_{3}$ are explored as shown in Fig. 4 and listed in Table 1.

In power law model, we fix the parameter $\beta_{3}$ and find the values of other parameters $\beta_{1}$ and $\beta_{2}$. It is found that GSLT validate for the choice of $\beta_{3} \leq 0$ and the detailed results are shown in Table 2. In Fig. 5, we present graphical illustration of the validity range and show the evolution of GSLT versus $\beta_{3}$ for the choice $\beta_{1}=.02, \beta_{2}=0.3$ and $\sigma=2$.

In case of intermediate form, we have three parameters $\beta_{1}$, $\beta_{2}$ and $\beta_{3}$. We fix one parameter $\beta_{3}$ to set the validity ranges for the parameters $\beta_{1}$ and $\beta_{2}$ and results are shown in Table 2 . It is found that GSLT is valid only for $\beta \geq 0$, in case of $\beta \geq 0$ validity region exists only for earlier times. In this discussion, we find some cases where it is difficult to find valid regions. The graphical illustration of some cases is shown in Fig. 6, left plot shows the validity regions for $\beta_{3}=0$. It is found that GSLT is not valid for the choice of $\beta<0$ except some particular cases with specific regions. In the right plot of Fig. 6, we present the validity regions for $\beta_{3}=-2$. 

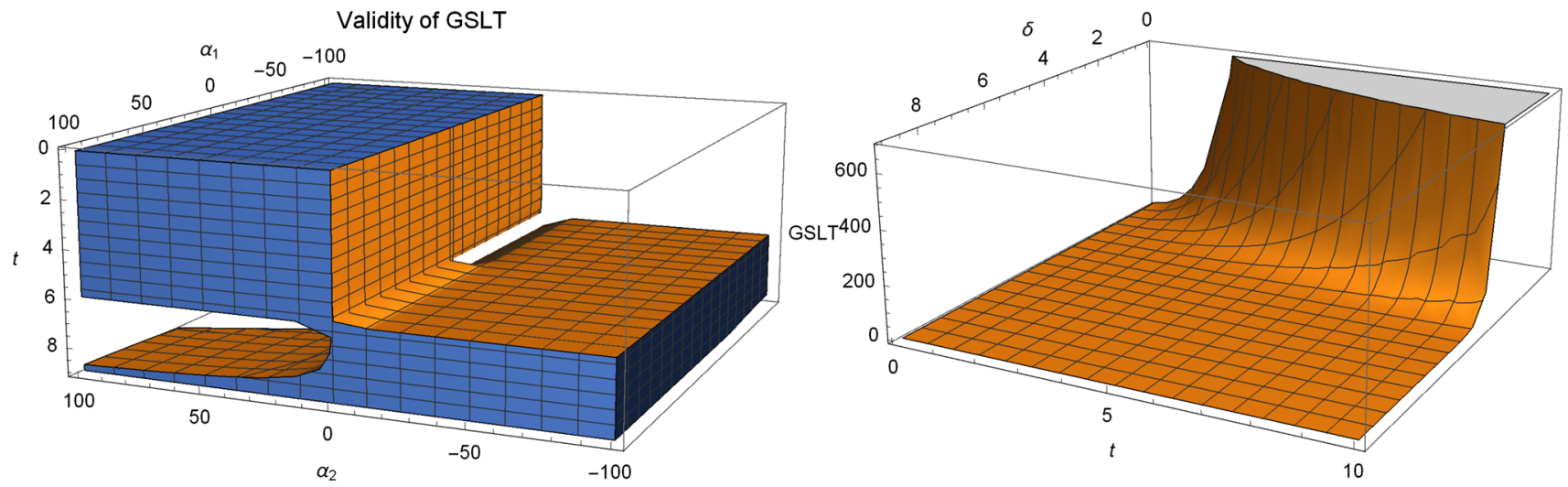

Fig. 2 Left plot represents the regions where GSLT is satisfied for $\delta=10$ and right graph corresponds to evolution of GSLT versus $\delta$ for $\alpha_{1}=-0.002, \alpha_{2}=0.001, b=2$ and $t_{s}=0.9$
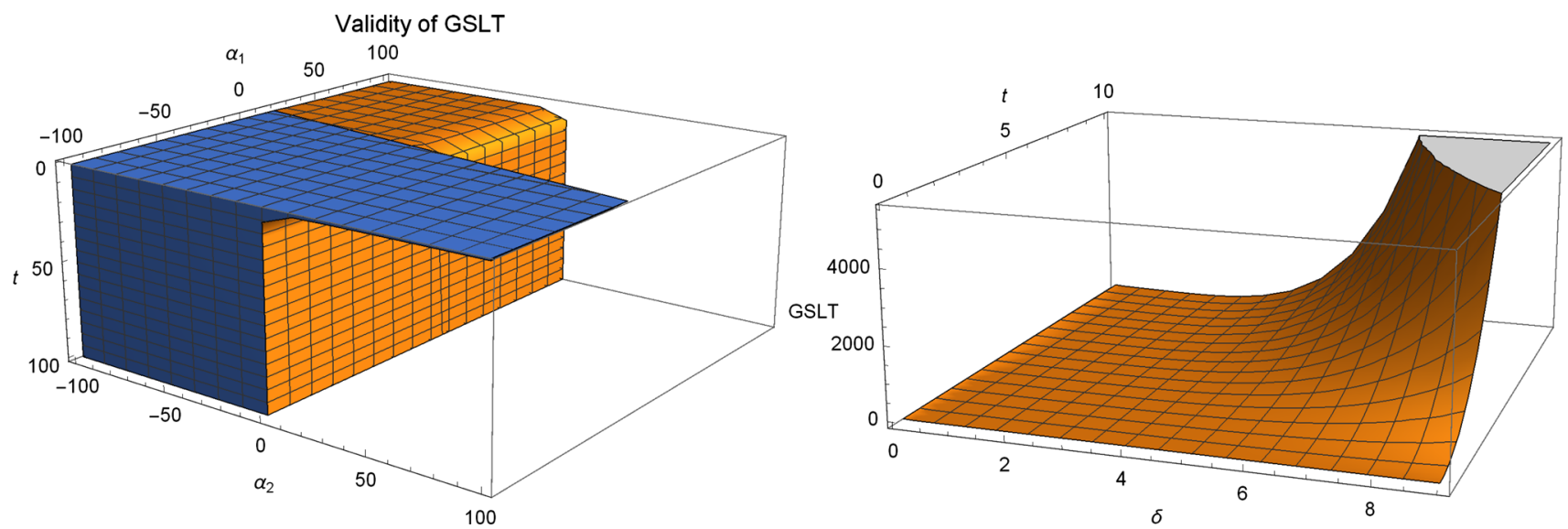

Fig. 3 Left plot represents the regions where GSLT is satisfied for $\delta=10$ in intermediate case and right graph corresponds to evolution of GSLT versus $\delta$ for $\alpha_{1}=0.2$ and $\alpha_{2}=-21$

\subsubsection{The validity of GSLT constraint for logarithmic corrected entropy}

Here we will consider the entropy correction formula involving logarithmic terms with effective gravitational coupling $\kappa_{e f f}^{2}$. In the present case, such entropy correction is defined by the following equation $[96,97]$

$\tilde{S}_{L C E}=\frac{A F_{T}}{4 G}+\lambda_{1} \ln \left(\frac{A F_{T}}{4 G}\right)+\lambda_{2}\left(\frac{4 G}{A F_{T}}\right)+\lambda_{3}$,

where $\lambda_{i} ; i=1,2,3$ are all non-zero dimensionless arbitrary constants. In case of Hubble horizon, the time rate of this entropy is given by

$$
\begin{aligned}
\dot{\tilde{S}}_{L C E}= & \frac{\pi}{G H^{2}}\left[1+\lambda_{1}\left(\frac{G H^{2}}{\pi F_{T}}\right)-\lambda_{2}\left(\frac{G H^{2}}{\pi F_{T}}\right)^{2}\right] \\
& \times\left(\dot{F}_{T}-\frac{2 \dot{H}}{H} F_{T}\right) .
\end{aligned}
$$

Consequently, the time rate of total entropy will become

$$
\begin{aligned}
\dot{\tilde{S}}_{t o t}= & \frac{4 \pi}{G} \frac{\dot{H}\left(\dot{H}+H^{2}\right) F_{T}}{\left(2 H^{2}+\dot{H}\right) H^{3}} \\
& +\frac{\pi}{G H^{2}}\left[1+\lambda_{1}\left(\frac{G H^{2}}{\pi F_{T}}\right)-\lambda_{2}\left(\frac{G H^{2}}{\pi F_{T}}\right)^{2}\right] \\
& \times\left(\dot{F}_{T}-\frac{2 \dot{H}}{H} F_{T}\right) \geq 0 .
\end{aligned}
$$

For the functional form defined by the Eq. (28), this constraint will take the form

$$
\begin{aligned}
\dot{\tilde{S}}_{t o t}= & \left\{\frac{4 \pi}{G} \frac{\dot{H}\left(\dot{H}+H^{2}\right)}{\left(2 H^{2}+\dot{H}\right) H^{3}}\right. \\
& \left.-\frac{2 \pi \dot{H}}{G H^{3}}\left(1+\lambda_{1}\left(\frac{G H^{2}}{\pi F_{T}}\right)-\lambda_{2}\left(\frac{G H^{2}}{\pi F_{T}}\right)^{2}\right)\right\} \\
& \times\left\{1-\frac{2 \alpha_{1} X_{1}}{T^{3}}-\frac{4 \alpha_{2} \delta X_{1}}{T^{5}} e^{\frac{\delta X_{1}}{T^{4}}}\right\}
\end{aligned}
$$




$$
\begin{aligned}
& +\frac{\pi}{G H^{2}}\left(1+\lambda_{1}\left(\frac{G H^{2}}{\pi F_{T}}\right)-\lambda_{2}\left(\frac{G H^{2}}{\pi F_{T}}\right)^{2}\right) \\
& \times\left\{\left(\frac{6 \alpha_{1} X_{1}}{T^{4}}+\left(\frac{16 \alpha_{2} \delta^{2} X_{1}^{2}}{T^{10}}+\frac{20 \alpha_{2} \delta X_{1}}{T^{6}}\right) e^{\frac{\delta X_{1}}{T^{4}}}\right) \dot{T}\right. \\
& \left.+\left(-\frac{2 \alpha_{1}}{T^{3}}-\frac{4 \alpha_{2} \delta}{T^{5}} e^{\frac{\delta X_{1}}{T^{4}}} \times\left(1+\frac{\delta}{T^{4}}\right)\right) \dot{X}_{1}\right\} \geq 0 .
\end{aligned}
$$

For the function $F$ form given by the Eq. (34), this GSLT inequality can be written as

$$
\begin{aligned}
\dot{\tilde{S}}_{t o t}= & \left\{\frac{4 \pi}{G} \frac{\dot{H}\left(\dot{H}+H^{2}\right)}{\left(2 H^{2}+\dot{H}\right) H^{3}}\right. \\
& \left.-\frac{2 \pi \dot{H}}{G H^{3}}\left(1+\lambda_{1}\left(\frac{G H^{2}}{\pi F_{T}}\right)-\lambda_{2}\left(\frac{G H^{2}}{\pi F_{T}}\right)\right)\right\} \\
& \times\left\{1-\frac{\beta_{1} X_{2}}{T^{2}}-\frac{3 \beta_{2} X_{2}^{2}}{T^{4}}-\frac{3 \sigma X_{2} \beta_{3}}{T^{4}} e^{\frac{\sigma X_{2}}{T^{3}}}\right\} \\
& +\frac{\pi}{G H^{2}}\left(1+\lambda_{1}\left(\frac{G H^{2}}{\pi F_{T}}\right)-\lambda_{2}\left(\frac{G H^{2}}{\pi F_{T}}\right)^{2}\right) \\
& \times\left\{\left(\frac{2 \beta_{1} X_{2}}{T^{3}}+\frac{12 \beta_{2} X_{2}^{2}}{T^{5}}\right.\right.
\end{aligned}
$$

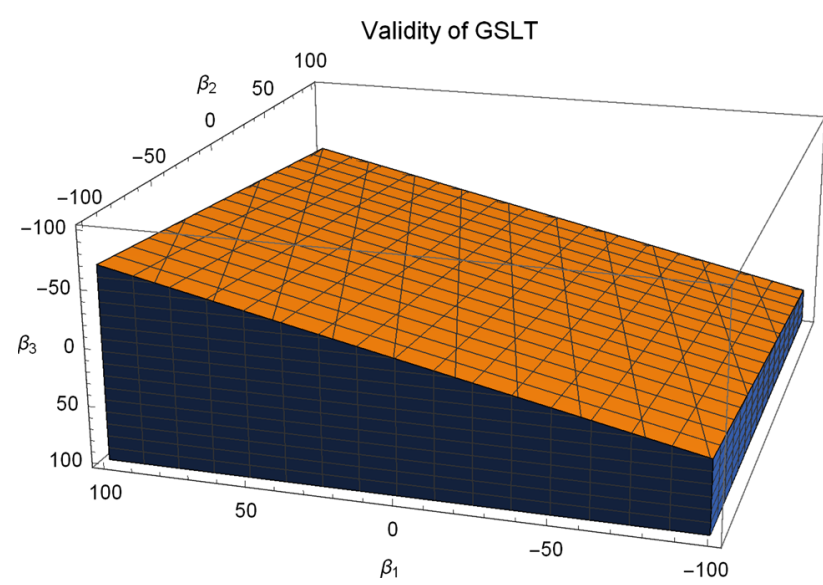

Fig. 4 The plot represents the validity regions for GSLT constraint in terms of cosmographic parameters for the model (34)

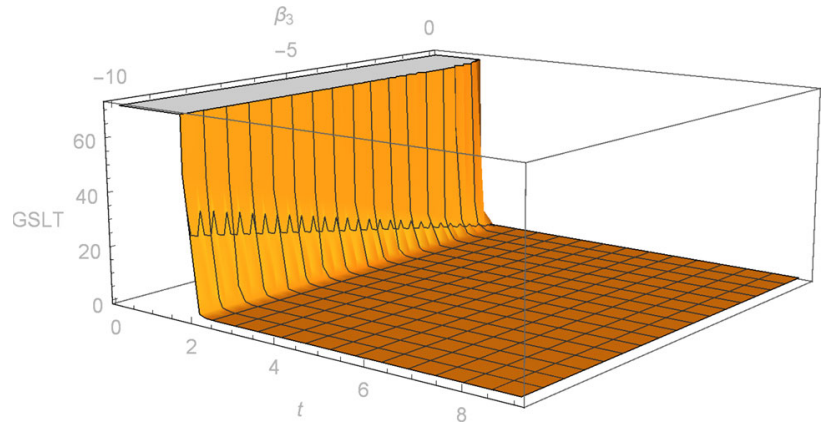

Fig. 5 The graphical illustration of GSLT versus $\beta_{3}$ for the second model. Herein, we set $\beta_{1}=.02, \beta_{2}=0.3$ and $\sigma=2$

$$
\begin{aligned}
& \left.+3 \sigma \beta_{3} X_{2}\left(\frac{4}{T^{5}}+\frac{3 \sigma X_{2}}{T^{4}}\right) e^{\frac{\sigma X_{2}}{T^{3}}}\right) \dot{T} \\
& \left.+\left(-\frac{\beta_{1}}{T^{2}}-\frac{6 \beta_{2} X_{2}}{T^{4}}-\frac{3 \sigma \beta_{3}}{T^{4}} e^{\frac{\sigma X_{2}}{T^{3}}}\left(1+\frac{\sigma}{T^{3}}\right)\right) \dot{X}_{2}\right\} \geq 0 .
\end{aligned}
$$

Using four cases for expansion factor and the corresponding terms, we will check the validity of these GSLT constraints given by Eqs. (38) and (40). In case of constant Hubble parameter $H_{0}$, the GSLT is satisfied if $-16.8-2.78 \lambda_{1}+$ $0.46 \lambda_{2} \geq 0$. Here we find a relation for the validity of GSLT which depends on two dimensionless parameters $\lambda_{1}$ and $\lambda_{2}$. We show the evolution of GSLT in Fig. 7. For the cosmographic parameters, the GSLT validity regions are explored in Fig. 8. Here the left and right plots correspond to the GSLT constraints (38) and (40), respectively. The detail possible ranges of free model parameters $\alpha_{1}, \alpha_{2}, \delta, \beta_{1}, \beta_{2}$ and $\beta_{3}$ for which GSLT conditions remain valid are listed in Table 1.

Next we search for validity regions in case of power law model and the results are presented depending on different values of $\delta$. Some validity regions are presented in Fig. 9 for both $\delta=2$ and $\delta=-2$ respectively. In case of intermediate form of scale factor, we show the validity regions of GSLT for two cases $\delta=0$ and $\delta>0$. Left plot of Fig. 10 shows some particular validity regions for $\delta=0$ whereas in right plot we select $\delta>0$. The results of validity regions are shown in Table 2.

We also examine the validity of GSLT constraint for logarithmic corrected entropy in the background of function independent of $X_{1}$. It is found that GSLT is trivially satisfied for de Sitter model. In this case, we fix $\beta_{3}$ and develop the validity regions depending on the values of $\beta_{1}$ and $\beta_{2}$ for both power law and intermediate cases. In intermediate form particular validity regions are shown in Fig. 11 and other validity ranges can be seen in Table 2.

\section{Equilibrium picture}

Here we will talk about the equilibrium picture of first and generalized second law of thermodynamics. Here we consider the field equations as

$$
\begin{aligned}
& 6 F_{T} H^{2}+\left(24 H^{2} F_{X_{1}}+F_{X_{2}}\right)(3 H \dot{H}+\ddot{H}) H \\
& \quad+F_{X_{2}} \dot{H}^{2}+\left(3 H^{2}-\dot{H}\right) H \dot{F}_{X_{2}} \\
& \quad+24 H^{3} \dot{H} \dot{F}_{X_{1}}+H^{2} \ddot{F}_{X_{2}}+\frac{F}{12}=\rho_{m}, \\
& 2\left(F_{T} \dot{H}+H \dot{F}_{T}+24 H\left[2 H \ddot{H}+3\left(\dot{H}+H^{2}\right) \dot{H}\right] \dot{F}_{X_{1}}\right. \\
& \quad+12 H \dot{H} \dot{F}_{X_{2}}+24 H^{2} \dot{H} \ddot{F}_{X_{1}} \\
& \quad+\left(\dot{H}+3 H^{2}\right) \ddot{F}_{X_{2}}+24 H^{2} F_{X_{1}} \dddot{H}+H \dddot{F}_{X_{2}} \\
& \quad+24 F_{X_{1}} \dot{H}^{2}\left(12 H^{2}+\dot{H}\right) \\
& \left.\quad+24 H F_{X_{1}}\left(4 \dot{H}+3 H^{2}\right) \ddot{H}\right)=-p_{m} .
\end{aligned}
$$



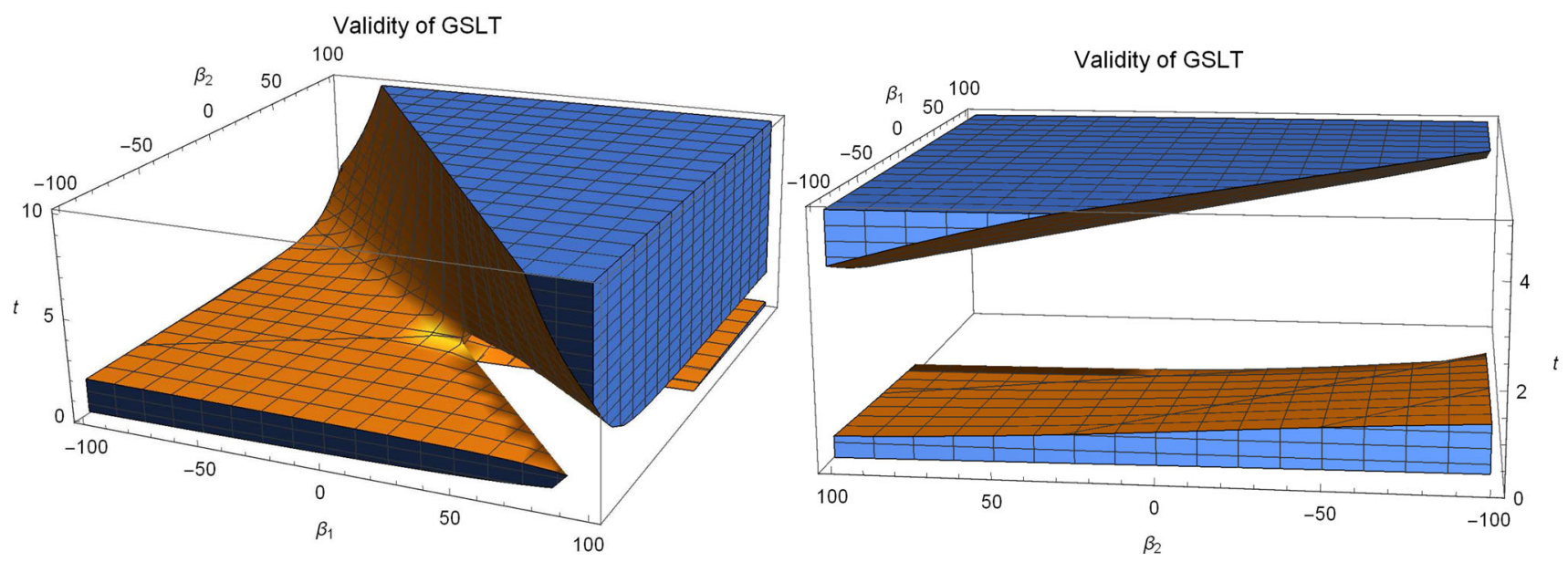

Fig. 6 Left plot represents the regions where GSLT is satisfied for $\beta_{3}=0$ in intermediate case and right graph corresponds to evolution of GSLT versus $\beta_{3}=-2$. Herein, we set $b_{1}=2, \sigma=2$ and $\beta=0.5$

It is worthwhile to mention here that all the discussion about first law of thermodynamics as presented in Sect. 3 is same except there is no entropy production term. It is due to the fact that there is no effective coupling term $\kappa_{\text {eff }}$ defined in the FRW field equations and consequently, the usual energy conservation equation for both ordinary matter as well as energy and pressure due to torsion scalar remain satisfied. Also, one need to use the usual form of Bekenstein-Hawking entropy relationship given by $S=\frac{A}{4 G}$ instead of its modified version. Thus, in this case, the first law of thermodynamics takes the form

$$
T_{A} d \tilde{S}_{A}=-d E+d W
$$

The relationship between entropy due to matter and energy sources inside the horizon $S_{i n}$ and the density and pressure in the horizon as provided by Gibb's equation can be written as

$$
T_{i n} d \tilde{S}_{i n}=d\left(\rho_{m} V\right)+p_{m} d V
$$

which can also be expressed as

$$
T_{i n} \dot{\tilde{S}}_{i n}=4 \pi \tilde{r}_{A}^{2}\left(\rho_{m}+p_{m}\right)\left(\dot{\tilde{r}}_{A}-H \tilde{r}_{A}\right)
$$

Using the relations for $\rho_{m}$ and $p_{m}$ computed from Eqs. (41) and (42), we get the GSLT in the following form

$$
\begin{aligned}
T_{A} \dot{\tilde{S}}_{t o t}= & -\frac{\dot{H}}{2 G H^{4}}\left(2 H^{2}+\dot{H}\right) \\
& -\frac{2 \pi \kappa^{2}}{H^{4}}\left(H^{2}+\dot{H}\right)\left[6 F_{T} H^{2}+6\left(24 H^{2} F_{X_{1}}\right.\right. \\
& \left.+F_{X_{2}}\right)(3 H \dot{H}+\ddot{H}) H+6 F_{X_{2}} \dot{H}^{2} \\
& +18 H^{3} \dot{F}_{X_{2}}-6 H \dot{H} \dot{F}_{X_{2}}+144 H^{3} \dot{H} \dot{F}_{X_{1}}
\end{aligned}
$$

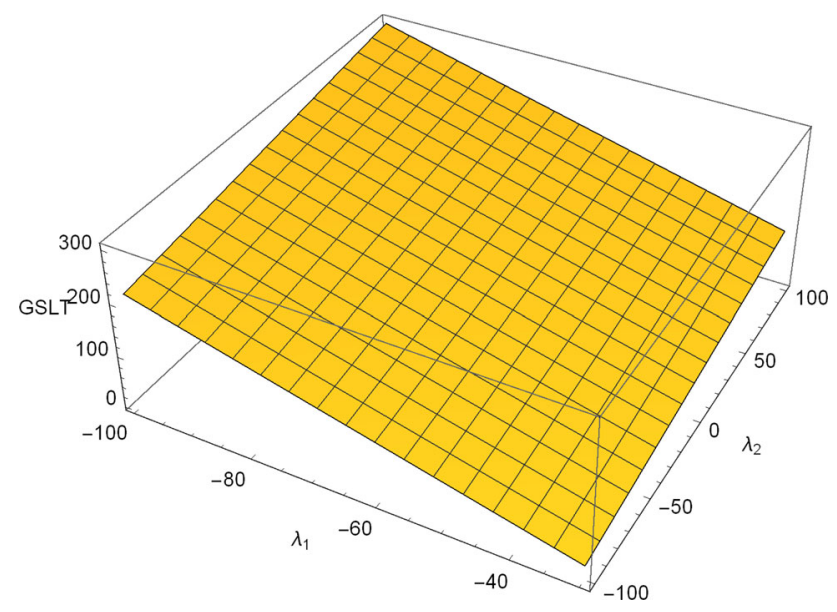

Fig. 7 Validity of GSLT for logarithmic corrected entropy with constant Hubble parameter

$$
\begin{aligned}
& +6 H^{2} \ddot{F}_{X_{2}}+\frac{F}{2}-2 F_{T} \dot{H}-2 H \dot{F}_{T} \\
& -48 H\left\{2 H \ddot{H}+3\left(\dot{H}+H^{2}\right) \dot{H}\right\} \dot{F}_{X_{1}} \\
& -24 H \dot{H} \dot{F}_{X_{2}}-48 H^{2} \dot{H} \ddot{F}_{X_{1}}-2\left(\dot{H}+3 H^{2}\right) \ddot{F}_{X_{2}} \\
& -48 H^{2} F_{X_{1}} \dddot{H}-2 H \dddot{F}_{X_{2}}-48 F_{X_{1}} \dot{H}^{2}\left(12 H^{2}+\dot{H}\right) \\
& \left.-48 H F_{X_{1}}\left(4 \dot{H}+3 H^{2}\right) \ddot{H}\right] \geq 0 .
\end{aligned}
$$

The derivative terms present in this constraint will be evaluated by using chain rule as follows:

$$
\begin{aligned}
\dot{F}_{X_{1}}= & F_{X_{1} T} \dot{T}+F_{X_{1} X_{1}} \dot{X}_{1}+F_{X_{1} X_{2}} \dot{X}_{2}, \\
\dot{F}_{X_{2}}= & F_{X_{2} T} \dot{T}+F_{X_{2} X_{1}} \dot{X}_{1}+F_{X_{2} X_{2}} \dot{X}_{2}, \\
\ddot{F}_{X_{1}}= & F_{X_{1} T T} \dot{T}^{2}+2 F_{X_{1} X_{1} T} \dot{X}_{1} \dot{T} \\
& +2 F_{T X_{1} X_{2}} \dot{T} \dot{X}_{2}+F_{X_{1} T} \ddot{T}+F_{X_{1} X_{1} X_{1}} \dot{X}_{1}^{2} \\
& +2 F_{X_{1} X_{1} X_{2}} \dot{X}_{1} \dot{X}_{2}+F_{X_{1} X_{1}} \ddot{X}_{1}
\end{aligned}
$$



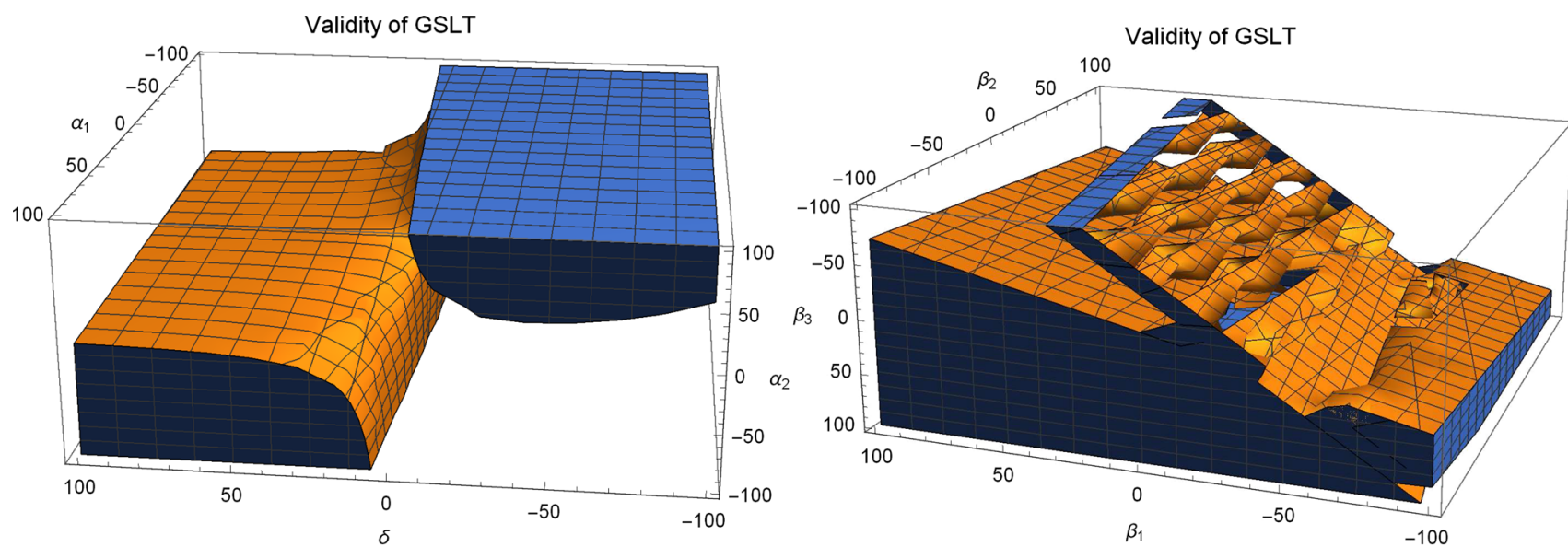

Fig. 8 Left and right plots represent the possible validity regions for logarithmic corrected entropy in terms of cosmographic parameters for both $F$ models
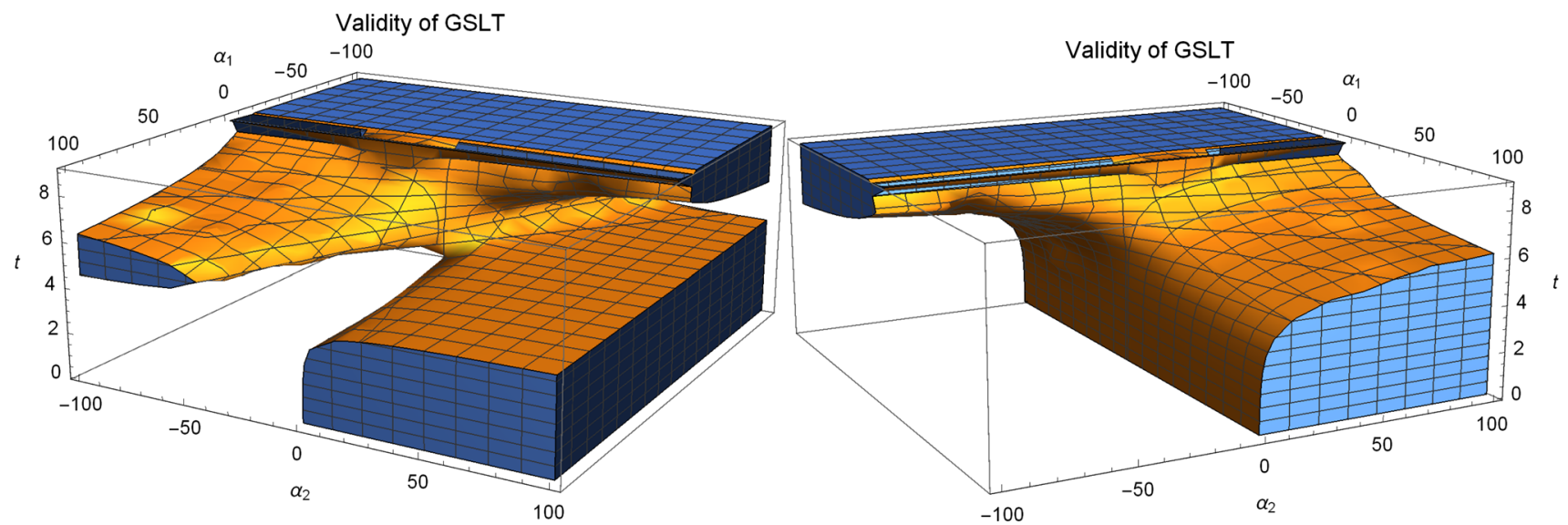

Fig. 9 Left plot represents the validity regions for logarithmic corrected entropy in power law case with $\delta=2$, right graph corresponds to evolution of GSLT for $\delta=-2$. Herein, we set $\lambda_{1}=2$ and $\lambda_{2}=3$

$$
\begin{aligned}
& +F_{X_{1} X_{2} X_{2}} \dot{X}_{2}^{2}+F_{X_{1} X_{2}} \ddot{X}_{2}, \\
& \ddot{F}_{X_{2}}=F_{X_{2} T T} \dot{T}^{2}+2 F_{X_{2} X_{1} T} \dot{X}_{1} \dot{T} \\
& +2 F_{T X_{2} X_{2}} \dot{T} \dot{X}_{2}+F_{X_{2} T} \ddot{T}+F_{X_{2} X_{1} X_{1}} \dot{X}_{1}^{2} \\
& +2 F_{X_{2} X_{1} X_{2}} \dot{X}_{1} \dot{X}_{2}+F_{X_{2} X_{2}} \ddot{X}_{2} \\
& +F_{X_{2} X_{2} X_{2}} \dot{X}_{2}^{2}+F_{X_{2} X_{2}} \ddot{X}_{2} \text {, } \\
& \dddot{F}_{X_{2}}=F_{X_{2} T T T} \dot{T}^{3}+3 F_{X_{2} T T X_{1}} \dot{X}_{1} \dot{T}^{2} \\
& +3 F_{X_{2} X_{2} T T} \dot{X}_{2} \dot{T}^{2}+3 \dot{T} \ddot{T} F_{X_{2} T T} \\
& +3 F_{X_{2} T X_{1} X_{1}} \dot{X}_{1}^{2} \dot{T}+F_{X_{2} T X_{1}}\left(\ddot{X}_{1} \dot{T}+\dot{X}_{1} \ddot{T}\right) \\
& +F_{X_{2} T X_{2}}\left(\ddot{X}_{2} \dot{T}+\dot{X}_{2} \ddot{T}\right) \\
& +3 F_{X_{2} X_{2} X_{2} T} \dot{X}_{2}^{2} \dot{T}+F_{X_{2} T X_{1}} \dot{X}_{1} \ddot{T} \\
& +F_{X_{2} T X_{2}} \dot{X}_{2} \ddot{T}+F_{X_{2} T} \dddot{T} \\
& +3 F_{X_{2} X_{1} T X_{2}} \dot{T} \dot{X}_{1} \dot{X}_{2}+F_{X_{2} X_{1} T}\left(\ddot{T} \dot{X}_{1}+\dot{T} \ddot{X}_{1}\right) \\
& +F_{X_{2} X_{1} X_{1} X_{1}} \dot{X}_{1}^{3} \\
& +F_{X_{2} X_{1} X_{1} X_{2}} \dot{X}_{1}^{2} \dot{X}_{2}+F_{X_{2} X_{1} X_{2} X_{2}} \dot{X}_{1} \dot{X}_{2}^{2}
\end{aligned}
$$

$$
\begin{aligned}
& +F_{X_{2} X_{1} X_{2}}\left(\ddot{X}_{1} \dot{X}_{2}+\dot{X}_{1} \ddot{X}_{2}\right) \\
& +3 F_{X_{2} X_{1} X_{1}} \dot{X}_{1} \ddot{X}_{1}+F_{X_{2} X_{1} T} \dot{T} \ddot{X}_{1} \\
& +F_{X_{2} X_{1} X_{2}} \ddot{X}_{1} \dot{X}_{2}+F_{X_{2} X_{1}} \dddot{X}_{1} \\
& +F_{X_{2} X_{2} T}\left(\ddot{X}_{2} \dot{T}+\dot{X}_{2} \ddot{T}\right)+F_{X_{2} X_{1} X_{1} T} \dot{T} \dot{X}_{1} \dot{X}_{2} \\
& +F_{X_{2} X_{2} X_{1} X_{1}} \dot{X}_{1}^{2} \dot{X}_{2} \\
& +2 F_{X_{2} X_{2} X_{2} X_{1}} \dot{X}_{1} \dot{X}_{2}^{2}+F_{X_{2} X_{2} X_{1}}\left(\ddot{X}_{1} \dot{X}_{2}+\dot{X}_{1} \ddot{X}_{2}\right) \\
& +F_{X_{2} X_{2} X_{2} X_{2}} \dot{X}_{2}^{3} \\
& +3 F_{X_{2} X_{2} X_{2}} \dot{X}_{2} \ddot{X}_{2}+F_{X_{2} X_{2} T} \dot{T} \ddot{X}_{2} \\
& +F_{X_{2} X_{2} X_{1}} \dot{X}_{1} \ddot{X}_{2}+F_{X_{2} X_{2}} \dddot{X}_{2} \text {, } \\
& \dot{F}_{T}=F_{T T} \dot{T}+F_{T X_{1}} \dot{X}_{1}+F_{T X_{2}} \dot{X}_{2} \text {. }
\end{aligned}
$$

Here we will investigate the validity of GSLT given by constraint Eq. (45) in this generalized teleparallel gravity. For this purpose, we examine the compatibility of this constraint 
for two specific forms of generic function $F\left(T, X_{1}, X_{2}\right)$ in the upcoming subsections.

\subsection{The validity of GSLT for $F$ independent of $X_{2}$}

Here we will explore the validity of GSLT using the form of $F$ given by Eq. (28). The GSLT constraint (45), in this case, takes the following form

$$
\begin{aligned}
& T_{h} \dot{\tilde{S}}_{t o t}=-\frac{\dot{H}}{2 G H^{4}}\left(2 H^{2}+\dot{H}\right) \\
&-\frac{2 \pi \kappa^{2}}{H^{4}}\left(H^{2}+\dot{H}\right) \times\left[\left(2 \dot{H}-6 H^{2}\right)\right. \\
&+\left\{\frac{2 \alpha_{1} X_{1}}{T^{3}}+\frac{4 \delta \alpha_{2} X_{1}}{T^{5}} e^{\frac{\delta X_{1}}{T^{4}}}\right\} \\
&+\left(144 H^{3}(3 H \dot{H}+\ddot{H})-48 H \ddot{H}\left(4 \dot{H}+3 H^{2}\right)\right. \\
&\left.-48 H^{2} \dddot{H}-48 \dot{H}^{2}\left(12 H^{2}+\dot{H}\right)\right)\left\{\frac{\alpha_{1}}{T^{2}}+\frac{\alpha_{2} \delta}{T^{4}} e^{\frac{\delta X_{1}}{T^{4}}}\right\} \\
&+\left(144 H^{3} \dot{H}-48 H \times\left(2 H \ddot{H}+3 \dot{H}\left(\dot{H}+H^{2}\right)\right)\right) \\
&\left\{\left(-\frac{4 \alpha_{2} \delta^{2} X_{1}}{T^{9}}-\frac{4 \alpha_{2} \delta}{T^{5}}\right) \dot{T}+\left(\frac{\alpha_{2} \delta^{2}}{T^{8}} e^{\frac{\delta X_{1}}{T^{4}}}\right) \dot{X}_{1}\right\} \\
&+\frac{1}{2}\left(T+\frac{\alpha_{1} X_{1}}{T^{2}}+\alpha_{2} e^{\frac{\delta X_{1}}{T^{4}}}\right) \\
&-2 H\left\{\left(\frac{6 \alpha_{1} X_{1}}{T^{4}}+\frac{20 \delta X_{1} \alpha_{2}}{T^{6}} e^{\frac{\delta X_{1}}{T^{4}}}\right.\right. \\
&\left.+\frac{16 \alpha_{2} \delta^{2} X_{1}^{2}}{T^{10}} e^{\frac{\delta X_{1}}{T^{4}}}\right) \dot{T} \\
&-\left(\frac{4 \alpha_{2} \delta^{2} X_{1}}{T^{9}}+\frac{4 \alpha_{2} \delta}{T^{5}}\right) e^{\left.\frac{\delta X_{1}}{T^{4}} \dot{X}_{1}\right\}} \\
&-48 H^{2} \dot{H}\left\{\left(\frac{36 \alpha_{2} \delta^{2} X_{1}}{T^{10}}+\frac{20 \alpha_{2} \delta}{T^{6}}\right.\right. \\
&\left.+\frac{16 \alpha_{2} \delta^{3} X_{1}^{2}}{T^{14}}+\frac{16 \alpha_{2} \delta^{2} X_{1}}{T^{10}}\right) e^{\frac{\delta X_{1}}{T^{4}}} \dot{T}^{2} \\
&+2\left(-\frac{4 \alpha_{2} \delta^{2}}{T^{9}}+\frac{\delta}{T^{4}}\left(\frac{4 \alpha_{2} \delta^{2} X_{1}}{T^{9}}+\frac{4 \alpha_{2} \delta}{T^{5}}\right)\right) e^{\frac{\delta X_{1}}{T^{4}} \dot{X}_{1} \dot{T}} \\
&-\left(\frac{4 \alpha_{2} \delta^{2} X_{1}}{T^{9}}+\frac{4 \alpha_{2} \delta}{T^{5}}\right) e^{\frac{\delta X_{1}}{T^{4}}} \ddot{T} \\
&\left.\left.\left.e^{\frac{\delta X_{1}}{T^{4}}}\right) \dot{X}_{1}^{2}+\left(\frac{\alpha_{2} \delta^{2}}{T^{8}} e^{\frac{\delta X_{1}}{T^{4}}}\right) \ddot{X}_{1}\right\}\right] \geq 0 .
\end{aligned}
$$

Here we will investigate this GSLT condition validity for previously defined four different forms of expansion factor.

For the de Sitter case, it is seen that the validity of GSLT can be achieved for some particular choice of involved free parameter $\alpha_{i}$ as given in Table 1. Specifically, the GSLT condition is satisfied if $\alpha_{2}=3.11$. For the case of cosmographic parameters, some relevant useful derivative terms appearing in the constraint (52) can be expressed as

$$
\begin{aligned}
\dot{X}_{1} \dot{T} & \left.=-3456 H^{10}(1+q)^{2}\{1+q)^{2}+j+3 q+2\right\}, \\
\ddot{T} & =-12 H^{4}\left\{(1+q)^{2}+(j+3 q+2)\right\}, \\
\dddot{T} & =12 H^{5}\{3(1+q)(j+3 q+2)-(s-2 j-5 q-3)\}, \\
\ddot{X}_{1} & =288 H^{8}\left\{(1+q)^{4}+5(1+q)^{2}(j+3 q+2)\right.
\end{aligned}
$$

$$
\left.+(j+3 q+2)^{2}-(1+q)(s-2 j-5 q-3)\right\} .
$$

Using the recent values of cosmographic parameters, the higher-order time rates of the terms $T, X_{1}$ and $X_{2}$ attain the following values

$\ddot{H}=0.4072, \quad \dddot{H}=-0.5927, \quad \dddot{H}=16.1570$,

$\ddot{T}=-3.9214, \quad \dddot{T}=7.8266 \quad \ddot{X}_{1}=55.7851$.

By making the use of these values, the possible restrictions on free model parameters are represented in Fig. 12 and listed in Table 1.

Next we consider the possibility of power law form of expansion factor. The corresponding Hubble parameter, Torsion scalar and $X_{1}, X_{2}$ terms along with their derivative terms are given by (32). Some other required higher order time rates can be calculated as

$$
\begin{aligned}
\ddot{H} & =\frac{2 b}{\left(t_{s}-t\right)^{3}}, \quad \dddot{H}=\frac{6 b}{\left(t_{s}-t\right)^{4}}, \\
H^{(i v)} & =\frac{24 b}{\left(t_{s}-t\right)^{5}}, \quad H^{(v)}=\frac{120 b}{\left(t_{s}-t\right)^{6}}, \\
\ddot{X}_{1} & =\frac{6048 b^{4}}{\left(t_{s}-t\right)^{8}}, \quad \dot{X}_{1} \dot{T}=-\frac{10,368 b^{6}}{\left(t_{s}-t\right)^{10}}, \\
\ddot{T} & =-\frac{36 b^{2}}{\left(t_{s}-t\right)^{4}} .
\end{aligned}
$$

For the graphical analysis, the validity of GSLT constraint (52) is provided by the right part of Fig. 13.

In last, we will discuss the validity of the GSLT constraint using the intermediate form of expansion radius. For this form of expansion radius, the cosmological parameters like Hubble parameter, torsion scalar, terms $X_{1}, X_{2}$ and its first order time rates are given by (33). Some other higher order time derivatives required for the evaluation of GSLT constraint are

$$
\begin{aligned}
\ddot{H} & =b_{1} \beta(\beta-1)(\beta-2) t^{\beta-3}, \\
\dddot{H} & =b_{1} \beta(\beta-1)(\beta-2)(\beta-3) t^{\beta-4}, \\
H^{(i v)} & =b_{1} \beta(\beta-1)(\beta-2)(\beta-3)(\beta-4) t^{\beta-5}, \\
H^{(v)} & =b_{1} \beta(\beta-1)(\beta-2)(\beta-3)(\beta-4)(\beta-5) t^{\beta-6}, \\
\ddot{T} & =-12 b_{1}^{2} \beta^{2}(\beta-1)(2 \beta-3) t^{2 \beta-4}, \\
\ddot{X}_{1} & =288 b_{1}^{4} \beta^{4}(\beta-1)^{2}(2 \beta-3)(4 \beta-7) t^{4 \beta-8}, \\
\dot{X}_{1} \dot{T} & =-3456 b_{1}^{6} \beta^{6}(\beta-1)^{3}(2 \beta-3) t^{6 \beta-10} .
\end{aligned}
$$

In the similar fashion, we find the validity regions for intermediate cases in the framework of equilibrium picture. It is mentioned that for the case of $\delta>0$, GSLT is satisfied in later times in intermediate case. We also present some validity regions in Fig. 13. 

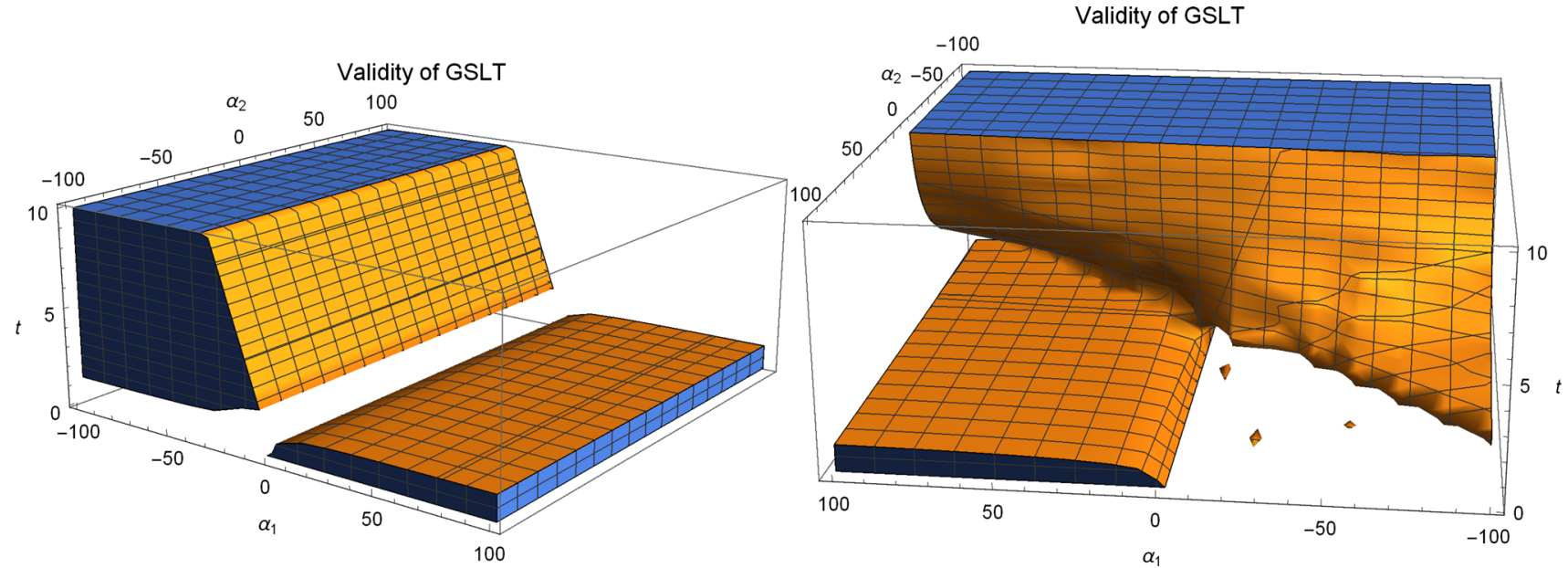

Fig. 10 Left plot represents the validity regions for logarithmic corrected entropy in intermediate case with $\delta=0$, right graph corresponds to evolution of GSLT for $\delta=2$. Herein, we set $\lambda_{1}=2$ and $\lambda_{2}=3$
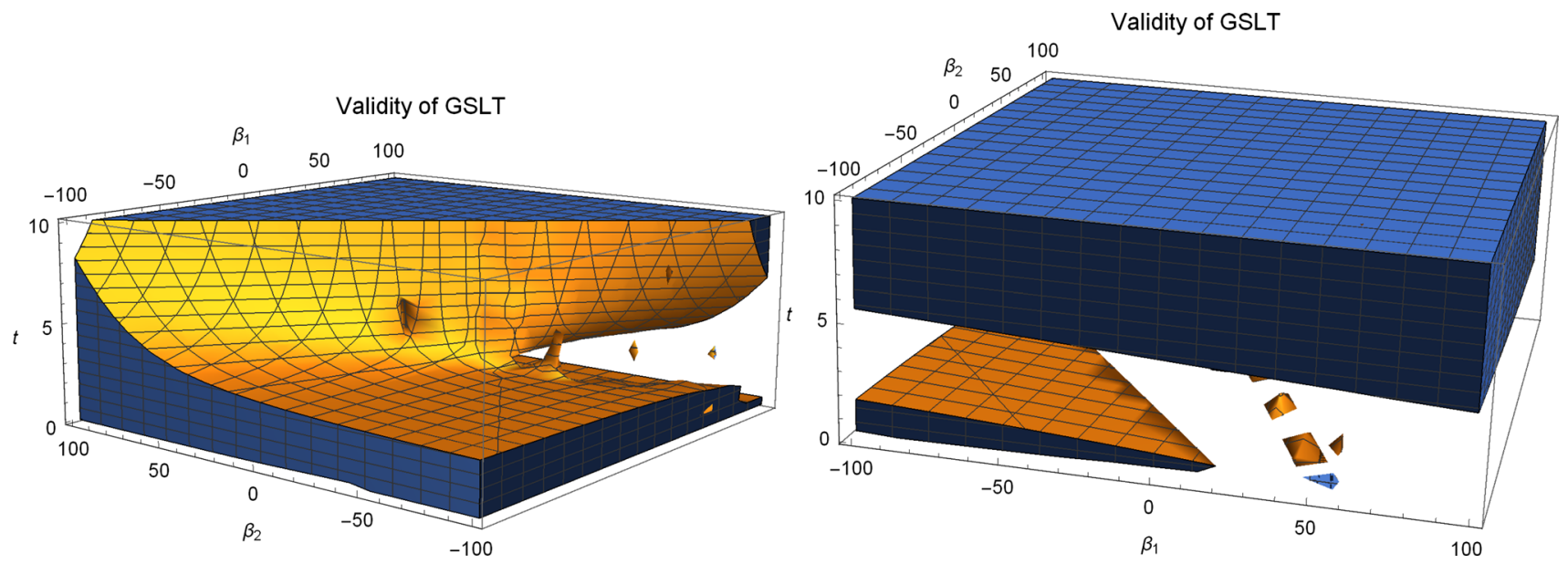

Fig. 11 Left plot represents the validity regions for logarithmic corrected entropy in intermediate case with $\beta_{3}=0$, right graph corresponds to evolution of GSLT for $\beta_{3}=-2$

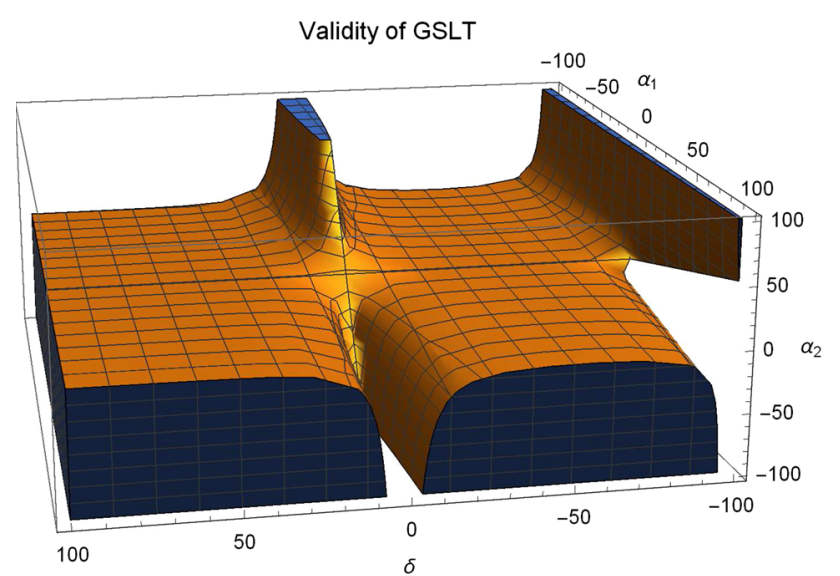

Fig. 12 Plot represents the validity regions of GSLT (52) for equilibrium case in terms of cosmographic parameters
4.2 The validity of GSLT for $F$ independent of $X_{1}$

Here we will consider the form of generic function of $F\left(T, X_{1}, X_{2}\right)$ given by Eq. (34). Removing the terms depending on $X_{1}$ and using the above defined form of $F$, the constraint for validity of GSLT is found as

$$
\begin{aligned}
T_{h} \dot{\tilde{S}}_{t o t}= & -\frac{\dot{H}}{2 G H^{4}}\left(2 H^{2}+\dot{H}\right) \\
& -\frac{2 \pi \kappa^{2}}{H^{4}}\left(H^{2}+\dot{H}\right)\left[\left(6 H^{2}-2 \dot{H}\right)\right. \\
& \times\left\{1-\frac{\beta X_{2}}{T^{2}}-\frac{3 \beta_{2} X_{2}^{2}}{T^{4}}-\frac{3 \sigma X_{2} \beta_{3}}{T^{4}} e^{\frac{\sigma X_{2}}{T^{3}}}\right\} \\
& +\left(6 H(3 H \dot{H}+\ddot{H})+6 \dot{H}^{2}\right) \\
& \times\left\{\frac{\beta_{1}}{T}+\frac{2 \beta X_{2}}{T^{3}}+\frac{\beta_{3} \sigma}{T^{3}} e^{\frac{\sigma X_{2}}{T^{3}}}\right\}
\end{aligned}
$$




$$
\begin{aligned}
& +\left(18 H^{3}-30 H \dot{H}\right)\left\{\left(-\frac{\beta_{1}}{T^{2}}-\frac{6 \beta_{2} X_{2}}{T^{4}}-e^{\frac{\sigma X_{2}}{T^{3}}}\right.\right. \\
& \left.\times\left(\frac{3 \beta_{3} \sigma^{2} X_{2}}{T^{7}}+\frac{3 \beta_{3} \sigma}{T^{4}}\right)\right) \dot{T} \\
& \left.+\left(\frac{2 \beta_{2}}{T^{3}}+\frac{\beta_{3} \sigma^{2}}{T^{6}} e^{\frac{\sigma X_{2}}{T^{3}}}\right) \dot{X}_{2}\right\} \\
& -2 \dot{H}\left\{\left(\frac{2 \beta_{1}}{T^{3}}+\frac{24 \beta_{2} X_{2}}{T^{5}}\right.\right. \\
& +e^{\frac{\sigma X_{2}}{T^{3}}}\left(\frac{21 \beta_{3} \sigma^{2} X_{2}}{T^{8}}+\frac{12 \beta_{3} \sigma}{T^{5}}\right) \\
& \left.\left.+e^{\frac{\sigma X_{2}}{T^{3}}}\left(\frac{3 \sigma X_{2}}{T^{4}}\right)\left(\frac{3 \beta_{3} \sigma^{2} X_{2}}{T^{7}}+\frac{3 \beta_{3} \sigma}{T^{4}}\right)\right) \dot{T}^{2}\right] \\
& +2 \dot{X}_{2} \dot{T}\left(-\frac{6 \beta_{2}}{T^{4}}-e^{\frac{\sigma X_{2}}{T^{3}}} \frac{3 \beta_{3} \sigma^{2}}{T^{7}}\right. \\
& \left.-e^{\frac{\sigma X_{2}}{T^{3}}}\left(\frac{3 \beta_{3} \sigma^{3} X_{2}}{T^{10}}+\frac{3 \beta_{3} \sigma^{2}}{T^{7}}\right)\right) \\
& +\left(-\frac{\beta_{1}}{T^{2}}-\frac{6 \beta_{2} X_{2}}{T^{4}}-e^{\frac{\sigma X_{2}}{T^{3}}}\left(\frac{3 \beta_{3} \sigma^{2} X_{2}}{T^{7}}+\frac{3 \beta_{3} \sigma}{T^{4}}\right)\right) \ddot{T} \\
& \left.+\left(\frac{\beta_{3} \sigma^{3}}{T^{9}} e^{\frac{\sigma X_{2}}{T^{3}}}\right) \dot{X}_{2}^{2}+\left(\frac{2 \beta_{2}}{T^{3}}+\frac{\beta_{3} \sigma^{2}}{T^{6}} e^{\frac{\sigma X_{2}}{T^{3}}}\right) \ddot{X}_{2}\right\} \\
& +\frac{1}{2}\left(T+\frac{\beta_{1} X_{2}}{T}+\frac{\beta_{2} X_{2}^{2}}{T^{3}}+\beta_{3} e^{\frac{\sigma X_{2}}{T^{3}}}\right) \\
& -2 H\left\{\left(\frac{2 \beta_{1} X_{2}}{T^{3}}+\frac{12 \beta_{2} X_{2}^{2}}{T^{5}}\right.\right. \\
& \left.+\frac{9 \sigma^{2} X_{2}^{2} \beta_{3}}{T^{8}} e^{\frac{\sigma X_{2}}{T^{3}}}+\frac{12 \sigma X_{2} \beta_{3}}{T^{5}} e^{\frac{\sigma X_{2}}{T^{3}}}\right) \dot{T} \\
& +\left(-\frac{\beta_{1}}{T^{2}}-\frac{6 \beta_{2} X_{2}}{T^{4}}\right. \\
& \left.\left.-e^{\frac{\sigma X_{2}}{T^{3}}}\left(\frac{3 \beta_{3} \sigma^{2} X_{2}}{T^{7}}+\frac{3 \beta_{3} \sigma}{T^{4}}\right)\right) \dot{X}_{2}\right\} \\
& -2 H\left\{\left(-\frac{6 \beta_{1}}{T^{4}}-\frac{120 \beta_{2} X_{2}}{T^{6}}-e^{\frac{\sigma X_{2}}{T^{3}}}\right.\right. \\
& \times\left(\frac{168 \beta_{3} \sigma^{2} X_{2}}{T^{9}}+\frac{60 \beta_{3} \sigma}{T^{6}}\right) \\
& -\frac{3 \sigma X_{2}}{T^{4}} e^{\frac{\sigma X_{2}}{T^{3}}}\left(\frac{21 \beta_{3} \sigma^{2} X_{2}}{T^{8}}+\frac{12 \beta_{3} \sigma}{T^{5}}\right)+e^{\frac{\sigma X_{2}}{T^{3}}} \\
& \times\left(\frac{3 \sigma X_{2}}{T^{4}}\right)^{2}\left(\frac{3 \beta_{3} \sigma^{2} X_{2}}{T^{7}}+\frac{3 \beta_{3} \sigma}{T^{4}}\right) \\
& \left.-e^{\frac{\sigma X_{2}}{T^{3}}}\left(3 \sigma X_{2}\right)\left(\frac{33 \beta_{3} \sigma^{2} X_{2}}{T^{12}}+\frac{24 \beta_{3} \sigma}{T^{9}}\right)\right) \dot{T}^{3} \\
& +3 \dot{X}_{2} \dot{T}^{2}\left(-\frac{3 \sigma^{3} \beta_{3}}{T^{10}} e^{\frac{\sigma X_{2}}{T^{3}}}\right. \\
& \left.-\frac{\sigma}{T^{3}}\left(\frac{6 \beta_{3} \sigma}{T^{7}}+\frac{3 \sigma^{3} \beta_{3} X_{2}}{T^{10}}\right) e^{\frac{\sigma X_{2}}{T^{3}}}\right) \\
& +3 \dot{T} \ddot{T}\left(\frac{2 \beta_{1}}{T^{3}}+\frac{24 \beta_{2} X_{2}}{T^{5}}\right. \\
& +e^{\frac{\sigma X_{2}}{T^{3}}}\left(\frac{21 \beta_{3} \sigma^{2} X_{2}}{T^{8}}+\frac{12 \beta_{3} \sigma}{T^{5}}\right)
\end{aligned}
$$

$$
\begin{aligned}
& \left.+e^{\frac{\sigma X_{2}}{T^{3}}}\left(\frac{3 \sigma X_{2}}{T^{4}}\right)\left(\frac{3 \beta_{3} \sigma^{2} X_{2}}{T^{7}}+\frac{3 \beta_{3} \sigma}{T^{4}}\right)\right) \\
& -3 \dot{X}_{2}^{2} \dot{T}\left(\frac{3 \sigma^{3} \beta_{3}}{T^{10}} e^{\frac{\sigma X_{2}}{T^{3}}}\right. \\
& \left.+\left(\frac{6 \beta_{3} \sigma}{T^{7}}+\frac{3 \sigma^{3} \beta_{3} X_{2}}{T^{10}}\right) \frac{\sigma}{T^{3}} e^{\frac{\sigma X_{2}}{T^{3}}}\right) \\
& +3\left(\ddot{X}_{2} \dot{T}+\dot{X}_{2} \ddot{T}\right)\left(-\frac{6 \beta_{2}}{T^{4}}-e^{\frac{\sigma X_{2}}{T^{3}}}\left(\frac{3 \beta_{3} \sigma^{2}}{T^{7}}\right)\right. \\
& \left.-e^{\frac{\sigma X_{2}}{T^{3}}} \frac{\sigma}{T^{3}}\left(\frac{3 \beta_{3} \sigma^{2} X_{2}}{T^{7}}+\frac{3 \beta_{3} \sigma}{T^{4}}\right)\right) \\
& +\left(-\frac{\beta_{1}}{T^{2}}-\frac{6 \beta_{2} X_{2}}{T^{4}}\right. \\
& \left.\left.-e^{\frac{\sigma X_{2}}{T^{3}}}\left(\frac{3 \beta_{3} \sigma^{2} X_{2}}{T^{7}}+\frac{3 \beta_{3} \sigma}{T^{4}}\right)\right) \dddot{T}\right) \\
& +\left(\frac{2 \beta_{2}}{T^{3}}+\frac{\beta_{3} \sigma^{2}}{T^{6}} e^{\frac{\sigma X_{2}}{T^{3}}}\right) \dddot{X}_{2} \\
& \left.\left.+3\left(\frac{\beta_{3} \sigma^{3}}{T^{9}} e^{\frac{\sigma X_{2}}{T^{3}}}\right) \dot{X}_{2} \ddot{X}_{2}+\frac{\beta_{3} \sigma^{4}}{T^{12}} e^{\frac{\sigma X_{2}}{T^{3}} \dot{X}_{2}^{3}}\right\}\right] \geq 0 .
\end{aligned}
$$

Using the same four choices of expansion radius, we will check the compatibility of this constraint graphically. It is seen that for de Sitter model, the GSLT constraint will be satisfied if we fix $\beta_{3}=-3.11$. For the case of cosmographic parameters, some useful higher order derivatives of the term $X_{2}$ are given by

$$
\begin{aligned}
\ddot{X}_{2}= & -12 H^{6}\left\{3(j+3 q+2)^{2}-4(1+q)(s-2 j-5 q-3)\right. \\
& +3(s-2 j-5 q-3)-18(1+q)(j+3 q+2) \\
& -6(1+q)^{3}+(l-5 s+10(q+2) j \\
& +30 q(q+2)+24)\}, \\
\dddot{X}_{2}= & -12 H^{7}\{10(j+3 q+2)(s-2 j-5 q-3) \\
& -5(1+q)(l-5 s+10(q+2) j+30 q(q+2)+24) \\
& +3(l-5 s+10(q+2) j+30 q(q+2) \\
& +24)-24(1+q)(s-2 j-5 q-3) \\
& \left.+36(1+q)^{2}(j+3 q+2)+18(j+3 q+2)^{2}\right\},
\end{aligned}
$$

which turn out to be as $\ddot{X}_{2}=-17.2684$ and $\dddot{X}_{2}=$ -50.7413 , for recent fix values of cosmographic quantities. In this case, the possible validity region for the GSLT constraint is given by Fig. 14 and the detail is provided in Table 1.

Also, for the power law form of scale factor, the higherorder time rates of $X_{2}$ turn out to be

$$
\begin{aligned}
\ddot{X}_{2} & =-\frac{720 b^{2}(1+b)}{\left(t_{s}-t\right)^{6}}, \quad \dddot{X}_{2}=-\frac{4320 b^{2}(1+b)}{\left(t_{s}-t\right)^{7}}, \\
\dot{X}_{2} \ddot{X}_{2} & =\frac{103680 b^{4}(1+b)^{2}}{\left(t_{s}-t\right)^{11}}, \quad \ddot{X}_{2} \dot{T}=\frac{8640 b^{4}(1+b)}{\left(t_{s}-t\right)^{9}}, \\
\dot{X}_{2} \ddot{T} & =\frac{5184 b^{4}(1+b)}{\left(t_{s}-t\right)^{9}} \quad \dot{X}_{2} \dot{T}=\frac{1728 b^{4}(1+b)^{2}}{\left(t_{s}-t\right)^{8}}
\end{aligned}
$$



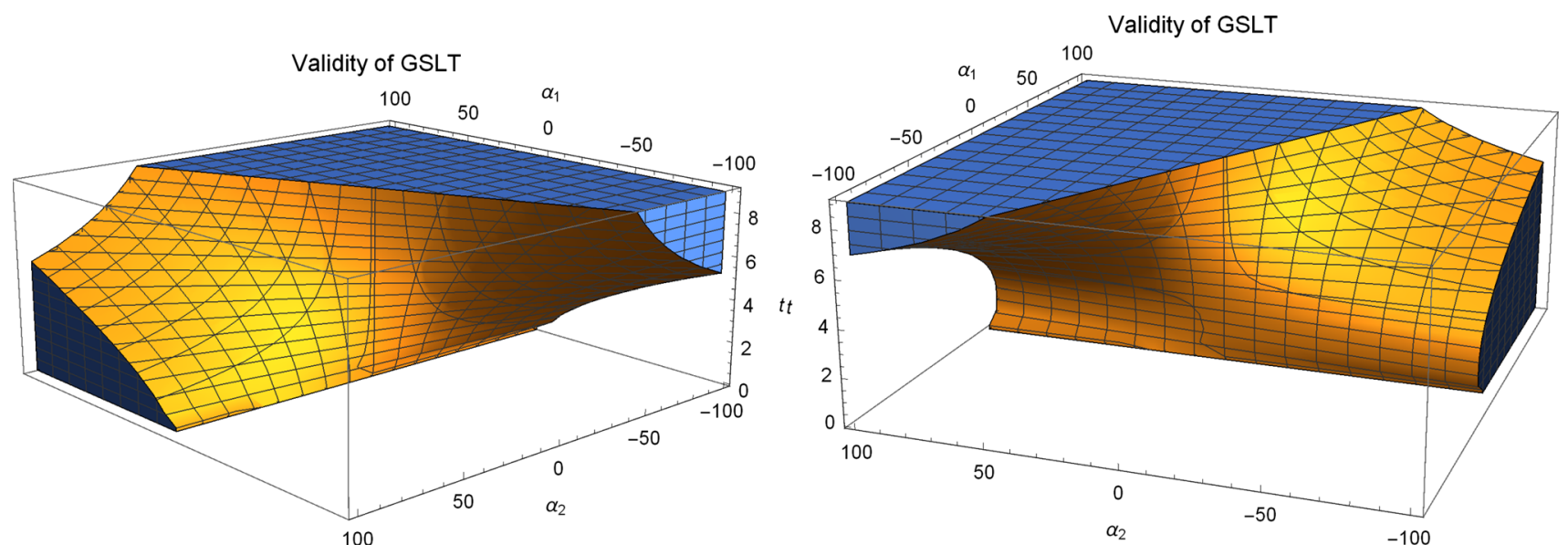

Fig. 13 Left plot represents the validity regions for logarithmic corrected entropy in intermediate case with $\delta=2$, right graph corresponds to evolution of GSLT for $\delta=-2$

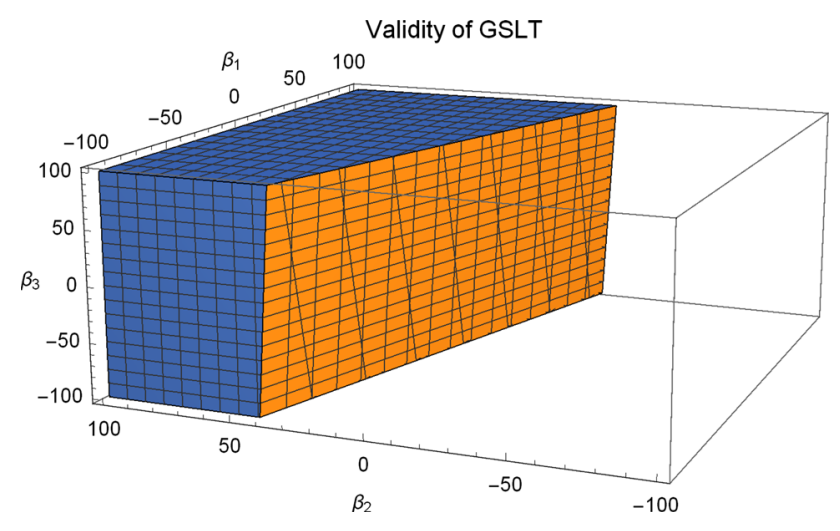

Fig. 14 Plot represents the validity regions for GSLT condition for equilibrium case in terms of cosmographic quantities for the model (34)

$$
\begin{aligned}
\dot{T} \ddot{T} & =\frac{432 b^{4}}{\left(t_{s}-t\right)^{4}}, \quad \dot{X}_{2}^{2} \dot{T}=-\frac{248832 b^{6}(1+b)^{2}}{\left(t_{s}-t\right)^{13}}, \\
\dot{X}_{2} \dot{T}^{2} & =-\frac{20736 b^{4}(1+b)}{\left(t_{s}-t\right)^{11}}
\end{aligned}
$$

Furthermore, for intermediate form of expansion factor, these derivatives are computed as follows

$$
\begin{aligned}
\ddot{X}_{2}= & -12\left\{b_{1}^{2} \beta^{2}(\beta-1)(2 \beta-3)(2 \beta-4)(2 \beta-5) t^{2 \beta-6}\right. \\
& \left.+3 b_{1}^{3} \beta^{3}(\beta-1) \times(3 \beta-4)(3 \beta-5) t^{3 \beta-6}\right\} \\
\dddot{X}_{2}= & -12\left\{b_{1}^{2} \beta^{2}(\beta-1)(2 \beta-3)(2 \beta-4)\right. \\
& \times(2 \beta-5)(2 \beta-6) t^{2 \beta-7}+3 b_{1}^{3} \beta^{3} \\
& \left.\times(\beta-1)(3 \beta-4)(3 \beta-5)(3 \beta-6) t^{3 \beta-7}\right\}
\end{aligned}
$$

Introducing these derivatives in the GSLT constraint (56), we check its validity by making graphical analysis as presented in Fig. 15. Here, in the left plot, we show the validity regions for $\beta_{3}=0$, while the right plot indicates the regions for $\beta_{3}=-2$. In case of $\beta_{3} \leq 0$, we can not find one particu- lar region of validity, in fact, there are very small regions as shown in this plot.

\section{Concluding remarks}

In the present manuscript, we have discussed the laws of thermodynamics in a generalized gravitational framework based on higher-order derivatives of torsion scalar. By taking flat FRW model with barotropic fluid as matter distribution, we have discussed the FLT and GSLT at Hubble horizon in both equilibrium and non-equilibrium perspectives. Firstly, we have presented the non-equilibrium picture of these thermodynamical laws in such gravity at the Hubble horizon of FRW model. In order to investigate the validity of resulting inequalities, we have used two specific models of $F\left(T, X_{1}, X_{2}\right)$ function and some interesting cases for scale factor namely, constant Hubble parameter, cosmographic parameters, power law and intermediate forms. In the same section, we have explored the validity of GSLT by taking logarithmic corrected entropy into account. In all cases, we have checked the validity of GSLT constraints graphically and found the possible conditions on the involved free model parameters.

In this generalized teleparallel gravity, it is seen that the gravitational equations can lead to the non-equilibrium picture of thermodynamical laws due to the presence of an extra entropy production term based on the function $F\left(T, X_{1}, X_{2}\right)$. This is quite similar to the cases of many other modified gravity theories like where such extra term appeared in FLT of thermodynamics ([80,87,88,95] etc.). In this nonequilibrium picture of thermodynamical laws, we investigated the validity of GSLT constraint using two models of $F\left(T, X_{1}, X_{2}\right)$ function both involving inverse and exponential torsion scalar terms. In the first place, by fixing some of 

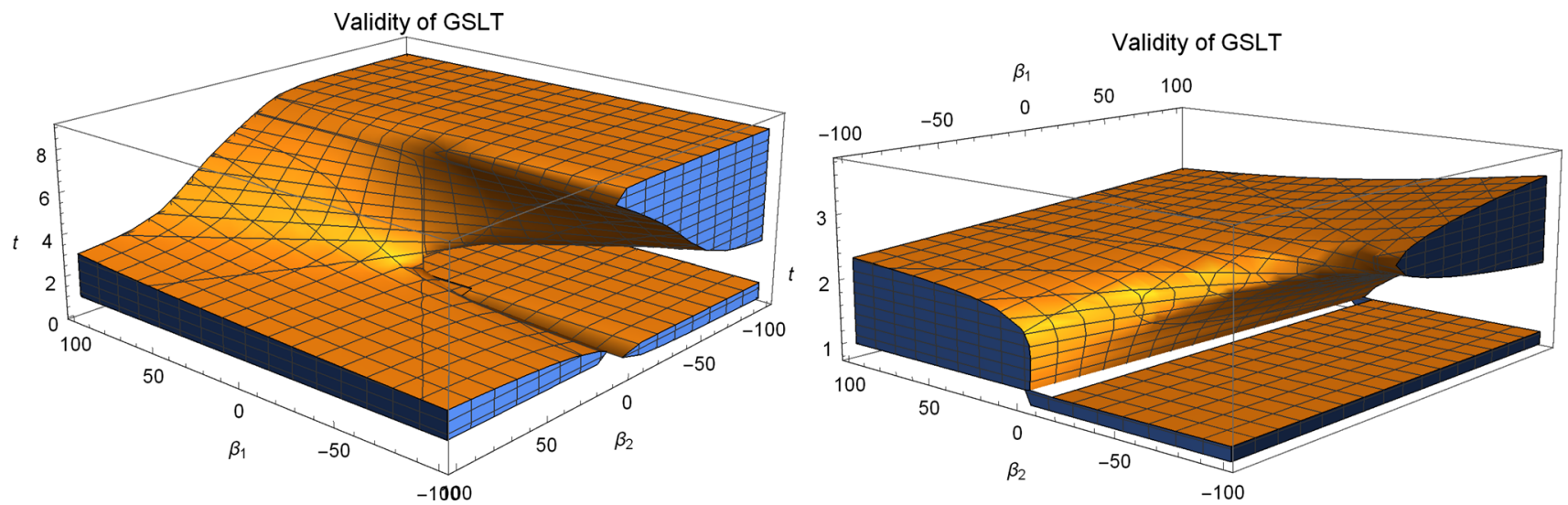

Fig. 15 Left plot represents the validity regions for equilibrium picture in intermediate case with $\beta_{3}=0$, right graph corresponds to evolution of GSLT for $\beta_{3}=-2$

the involved free model parameters, we explored the ranges of other parameters for which GSLT constraint remains satisfied using 3D region plots. Then by taking these interesting ranges of free parameters into account, we have shown the validity of GSLT graphically in few cases. We have also investigated the possible ranges of free parameters for the validity of GSLT constraints in the presence of logarithmic corrections in entropy relation using region graphs for both $F\left(T, X_{1}, X_{2}\right)$ models.

Furthermore, we have investigated the possibility of equilibrium picture existence of these thermodynamical laws. For previously used two models of the function $F\left(T, X_{1}, X_{2}\right)$, we formulated the resulting GSLT constraints at Hubble horizon and checked their validity using cosmographic parameters as well as the power and intermediate forms of expansion radius. A detailed graphical analysis of these inequalities and the possible restrictions on free parameters in terms of region graphs have also been presented there. All the possible constraints on the free parameters in both equilibrium as well as non-equilibrium perspectives in all cases of expansion radius can be summarized in the forms of Tables 1 and 2 .

In literature, this higher-order torsion derivatives based theory has only been investigated for stability analysis using fixed point theory and the validity of energy condition bounds for restricting free model parameters. In these discussions, a very limited analysis of free parameters selection has been provided. However, the present paper is providing a very detailed analysis of model parameters selection in order to make them compatible with the GSLT constraint and hence leading to a positive contribution in the regard. It would be worthwhile to explore the validity of GSLT constraints at apparent as well as event horizons in this generalized teleparallel gravity by constraining the free involved parameters.

Data Availability Statement This manuscript has no associated data or the data will not be deposited. [Authors' comment: We have introduced analytical results and observational data is yet to be used in further analysis in future work.]
Open Access This article is distributed under the terms of the Creative Commons Attribution 4.0 International License (http://creativecomm ons.org/licenses/by/4.0/), which permits unrestricted use, distribution, and reproduction in any medium, provided you give appropriate credit to the original author(s) and the source, provide a link to the Creative Commons license, and indicate if changes were made. Funded by SCOAP ${ }^{3}$.

\section{References}

1. S. Perlmutter et al., Nature 391, 51 (1998)

2. A.G. Riess et al., Astrophys. J. 116, 1009 (1998)

3. C.L. Bennett et al., Astrophys. J. Suppl. 148, 1 (2003)

4. S.W. Allen, R.W. Schmidt, H. Ebeling, A.C. Fabian, L.V. Speybroeck, Mon. Not. R. Astron. Soc. 353, 457 (2004)

5. M. Tegmark et al., Phys. Rev. D 69, 03501 (2004)

6. B. Ratra, P.J.E. Peebles, Phys. Rev. D 37, 3406 (1988)

7. V. Gorini, A.Y. Kamenshchik, U. Moschella, V. Pasquier, Phys. Rev. D 69, 123512 (2004)

8. T. Padmanabhan, Gen. Relativ. Gravit. 40, 529 (2008)

9. S. Chaplygin, Sci. Mem. Moscow Univ. Math. Phys. 21, 1 (1904)

10. M.C. Bento, O. Bertolami, A.A. Sen, Phys. Rev. D 66, 043507 (2002)

11. M. Sharif, M. Zubair, Int. J. Mod. Phys. D 19, 1957-1972 (2010)

12. M. Zubair, Adv. High Energy Phys. 2015, 292767 (2015)

13. F.S.N. Lobo, The dark side of gravity: modified theories of gravity, invited chapter to appear in an edited collection "dark energycurrent advances and ideas". arXiv:0807.1640

14. K. Bamba, S. Capozziello, S. Nojiri, S.D. Odintsov, Astrophys. Space Sci. 345, 155 (2012)

15. A. De Felice, S. Tsujikawa, Living Rev. Relativ. 13, 3 (2010)

16. S. Nojiri, S.D. Odintsov, Phys. Lett. B 631, 1 (2005)

17. G. Cognola et al., Phys. Rev. D 73, 084007 (2006)

18. K. Bamba, S.D. Odintsov, L. Sebastiani, S. Zerbini, Eur. Phys. J. C 67, 295 (2010)

19. M.E. Rodrigues, M.J.S. Houndjo, D. Momeni, R. Myrzakulov, Can. J. Phys. 92, 173 (2014)

20. S. Capozziello, S. Carloni, A. Troisi, Recent Res. Dev. Astron. Astrophys. 1, 625 (2003)

21. S. Nojiri, S.D. Odintsov, Phys. Rev. D 68, 123512 (2003)

22. S. Capozziello, V. Faraoni, Beyond Einstein Gravity: A Survey of Gravitational Theories for Cosmology and Astrophysics (Springer, New York, 2011) 
23. T. Harko, F.S.N. Lobo, S. Nojiri, S.D. Odintsov, Phys. Rev. D 84, 024020 (2011)

24. M.J.S. Houndjo, Int. J. Mod. Phys. D 21, 1250003 (2012)

25. F.G. Alvarenga, Phys. Rev. D 87, 103526 (2013)

26. M. Zubair, Hina Azmat, I. Noureen, Int. J. Mod. Phys. D 27, 1850047 (2018)

27. H. Azmat, M. Zubair, I. Noureen, Int. J. Mod. Phys. D 27, 1750181 (2017)

28. M. Zubair, G. Abbas, I. Noureen, Astrophys. Space Sci. 361, 8 (2016)

29. M. Zubair, M. Zeeshan, S.S. Hasan, V.K. Oikonomou, Symmetry 10, 463 (2018)

30. Z. Haghani et al., Phys. Rev. D 88, 044023 (2013)

31. S.D. Odintsov, D. Saez-Gomez, Phys. Lett. B 725, 437 (2013)

32. M. Sharif, M. Zubair, JCAP 11, 042 (2013)

33. M. Sharif, M. Zubair, JHEP 12, 079 (2013)

34. C.H. Brans, R.H. Dicke, Phys. Rev. 124, 925 (1961)

35. V. Faraoni, Cosmology in Scalar-Tensor Gravity (Kluwer Academic Publishers, Dordrecht, 2004)

36. M. Zubair, F. Kousar, Eur. Phys. J. C 76, 254 (2016)

37. M. Zubair, F. Kousar, S. Bahamonde, Phys. Dark Univ. 14, 116 (2016)

38. M. Zubair, F. Kousar, S. Bahamonde, Int. J. Mod. Phys. D 27, 1850115 (2018)

39. R. Ferraro, F. Fiorini, Phys. Rev. D 75, 084031 (2007)

40. E.V. Linder, Phys. Rev. D 81, 127301 (2010)

41. T. Wang, Phys. Rev. D 84, 024042 (2011)

42. S. Bahamonde, C.G. Böhmer, Eur. Phys. J. C 76(10), 578 (2016)

43. S. Bahamonde, S. Capozziello, Eur. Phys. J. C 77(2), 107 (2017)

44. M. Zubair, S. Waheed, Astrophys. Space Sci. 360, 68 (2015)

45. A. Einstein, Sitzungsber. Preuss. Akad. Wiss. Phys. Math. KI. 17, 217 (1928)

46. A. Einstein, Sitzungsber. Preuss. Akad. Wiss. Phys. Math. KI. 17, 224 (1928)

47. K. Hayashi, T. Shirafuji, Phys. Rev. D 19, 3524 (1979)

48. H.I. Arcos, J.G. Pereira, Int. J. Mod. Phys. D 13, 2193 (2004)

49. J.W. Maluf, Ann. Phys. 525, 339 (2013)

50. R. Aldrovandi, J.G. Pereira, Teleparallel Gravity: An Introduction (Springer, New York, 2013)

51. G. Kofinas, E.N. Saridakis, Phys. Rev. D 90, 084044 (2014)

52. G. Kofinas, E.N. Saridakis, Phys. Rev. D 90, 084045 (2014)

53. G. Kofinas, G. Leon, E.N. Saridakis, Class. Quantum Gravity 31, 175011 (2014)

54. Saira Waheed, M. Zubair, Astrophys. Space Sci. 359, 47 (2015)

55. T. Harko, F.S.N. Lobo, G. Otalora, E.N. Saridakis, Phys. Rev. D 89, 124036 (2014)

56. M. Zubair, S. Waheed, Astrophys. Space Sci. 355, 361 (2015)

57. S. Bahamonde, C.G. Bohmer, M. Wright, Phys. Rev. D 92, 104042 (2015)
58. S. Bahamonde, M. Zubair, G. Abbas, Phys. Dark Univ. 19, 78 (2018)

59. M. Zubair, S. Waheed, M.A. Fayyaz, I. Ahmad, Eur. Phys. J. Plus 133, 452 (2018)

60. G. Otalora, E.N. Saridakis, Phys. Rev. D 94, 084021 (2016)

61. S. Nojiri, S.D. Odintsov, Phys. Rev. D 505, 59 (2011)

62. S. Capozziello, M.D. Laurentis, Phys. Rep. 509, 167 (2011)

63. J.D. Bekenstein, Phys. Rev. D 7, 2333 (1973)

64. J.M. Bardeen, B. Carter, S.W. Hawking, Commun. Math. Phys. 31, $161(1973)$

65. S.W. Hawking, Commun. Math. Phys. 43, 199 (1975)

66. T. Jacobson, Phys. Rev. Lett. 75, 1260 (1995)

67. G.W. Gibbons, S.W. Hawking, Phys. Rev. D 15, 161 (1977)

68. A.V. Frolov, L. Kofman, J. Cosmol. Astropart. Phys. 05, 009 (2003)

69. T. Padmanabhan, Class. Quantum Gravity 19, 5387 (2002)

70. A. Sheykhi, B. Wang, R.G. Cai, Nucl. Phys. B 779, 1 (2007)

71. A. Sheykhi, B. Wang, R.G. Cai, Phys. Rev. D 76, 023515 (2007)

72. M. Akbar, R.G. Cai, Phys. Rev. D 75, 084003 (2007)

73. S.A. Hayward, Class. Quantum Gravity 15, 3147 (1998)

74. R. Cai, L. Cao, Y. Hu, S.P. Kim, Phys. Rev. D 78, 124012 (2008)

75. M. Akbar, R.G. Cai, Phys. Lett. B 648, 243 (2007)

76. K. Bamba, C.Q. Geng, JCAP 06, 014 (2010)

77. K. Bamba, C.Q. Genga, JCAP 11, 008 (2011)

78. R.G. Cai, L.M. Cao, Phys. Rev. D 75, 064008 (2007)

79. K. Karami, A. Abdolmaleki, J. Cosmo. Astropart. Phys. 1204, 007 (2012). (arXiv:1201.2511v2)

80. M. Sharif, M. Zubair, J. Cosmol. Astropart. Phys. 03, 028 (2012)

81. A. Abdolmaleki, T. Najafi, Int. J. Mod. Phys. D 25, 1650040 (2016)

82. H.M. Sadjadi, Europhys. Lett. 92, 50014 (2010)

83. M. Zubair, S. Bahamonde, M. Jamil, Eur. Phys. J. C 77, 472 (2017)

84. T. Azizi, N. Borhani, Adv. High Energy Phys. 2017, 6839050 (2017)

85. M. Zubair, A. Jawad, Astrophys. Space Sci. 360, 11 (2015)

86. M. Sharif, S. Waheed, Astrophys. Space Sci. 346, 583 (2013)

87. M. Sharif, S. Waheed, Astrophys. Space Sci. 349, 1003 (2013)

88. M. Sharif, M. Zubair, J. Cosmol. Astropart. Phys. 11, 042 (2013)

89. M. Sharif, M. Zubair, Adv. High Energy Phys. 2013, 947898 (2013)

90. T. Azizi, M. Gorjizadeh, arXiv:1701.00796v1

91. S. Capozziello, V.F. Cardone, H. Farajollahi, A. Ravanpak, Phys. Rev. D 84, 043527 (2011)

92. S. Nojiri, S.D. Odintsov, S. Tsujikawa, Phys. Rev. D 71, 063004 (2005)

93. H.M. Sadjadi, Phys. Rev. D 73, 063525 (2006)

94. J. Barrow, A. Rlidlle, C. Pahud, Phys. Rev. D 74, 127305 (2006)

95. M. Zubair, F. Kousar, S. Bahamonde, Phys. Dark Universe 14, 116 (2016)

96. M. Jamil, M.U. Farooq, J. Cosmol. Astropart. Phys. 3, 00 (2010)

97. H.M. Sadjadi, M. Jamil, Europhys. Lett. 92, 69001 (2010) 